Postprint: Vereecken E, Roels S, Janssen H, 2018. Inverse hygric property determination based on dynamic measurements and swarm-intelligence optimisers, Building and Environment 131: 184-196.

\title{
Inverse hygric property determination based on dynamic measurements and swarm-intelligence optimisers
}

\author{
EVY VEREECKENA* STAF ROELSB AND HANS JANSSENC
}

a KU Leuven, Department of Civil Engineering, Building Physics Section, Kasteelpark Arenberg 40, B-3001 Leuven, Belgium,

EVY.VEREECKEN@KULEUVEN.BE, TEL. +32 16321098, FAX +32 16321980

B KU Leuven, Department of Civil Engineering, Building Physics Section, Kasteelpark Arenberg 40, B-3001 Leuven, Belgium

STAF.ROELS@KULEUVEN.BE

C KU Leuven, Department of Civil Engineering, Building Physics Section, Kasteelpark Arenberg 40, B-3001 Leuven, Belgium

HANS.JANSSEN@KULEUVEN.BE

*CORRESPONDING AUTHOR 
Postprint: Vereecken E, Roels S, Janssen H, 2018. Inverse hygric property determination based on dynamic measurements and swarm-intelligence optimisers, Building and Environment 131: 184-196.

https://doi.org/10.1016/j.buildenv.2017.12.030

\section{Abstract}

To accelerate the hygric characterisation of porous building materials, dynamic flow and storage measurements in combination with inverse parameter estimation show a lot of promise. Therein though, the processing and interpretation of the experimental output can be challenging. This paper demonstrates the applicability of two swarmintelligence (SI) optimisers, i.e. the Particle Swarm Optimiser (PSO) and the Grey Wolf Optimiser (GWO), for determining the vapour resistance factor and the sorption isotherm of porous building materials. The methodology is presented for a fictitious dynamic vapour sorption experiment on a calcium silicate insulation sample. The identifiability of the unknown parameters and the reliability of the estimated properties is investigated via a profile likelihood (PL) analysis. By use of the proposed methodology, the measurement time required to determine the hygric properties is reduced strongly compared to standard techniques such as the steady-state cup and sorption tests, and at the same time the uncertainty propagated in the parameter estimation can be characterised. A close agreement with the target values is obtained. Though, to avoid an unreliable parameter estimation it is recommended to not limit the optimisation process to a single run. Furthermore, also the results obtained during the PL analysis can help improving the estimation. Finally, for the current case study, the SI optimisers are found to outperform the Genetic Algorithm (GA) and the Covariance Matrix Adaptation Evolution Strategy (CMA-ES). 
Postprint: Vereecken E, Roels S, Janssen H, 2018. Inverse hygric property determination based on dynamic measurements and swarm-intelligence optimisers, Building and Environment 131: 184-196.

https://doi.org/10.1016/j.buildenv.2017.12.030

\section{Key words:}

Hygric building properties; dynamic measurements; parameter estimation; Grey Wolf Optimiser; Particle Swarm Optimiser; identifiability

\section{Introduction}

Moisture transfer in building materials plays a vital role in the durability and sustainability of built structures $[1,2,3]$, and in the comfort and health of building occupants $[4,5,6]$. To properly assess existing constructions or correctly develop new constructions, the ability to analyse and predict the hygric performance of building materials is hence essential. This in turn requires knowledge of the hygric properties. The moisture storage and transport characteristics of the building materials are crucial input parameters in any building simulation model. Traditional measurement techniques - such as the steady-state cup or sorption tests [7,8] - applied to characterise these properties are however tedious and time-consuming. Dynamic measurements could accelerate the characterisation process; therein though the processing and interpretation of the experimental output is challenging.

Until recently, a deterministic inverse modeling approach making use of an appropriate analytical function was the common way to infer the material properties from a dynamic experimental output [9-15]. Such an approach, however, incurs limitations to the type of experiment and its boundary conditions and, furthermore, might imply a number of simplifications, among which the neglect of measurement noise and the assumption of perfectly imposed boundary conditions. These drawbacks can be eliminated by converting the determination process into an optimisation problem wherein the 
Postprint: Vereecken E, Roels S, Janssen H, 2018. Inverse hygric property determination based on dynamic measurements and swarm-intelligence optimisers, Building and Environment 131: 184-196.

https://doi.org/10.1016/j.buildenv.2017.12.030

difference between the experimental and modelled outputs are to be minimised. A deterministic gradient descent optimisation technique, such as the LevenbergMarquardt algorithm [16] can be applied to solve the - often non-linear - optimisation problem $[17,18,19]$. But, this method uses the Jacobian, which is often hard to calculate. Furthermore, the initial starting points applied in the algorithm can influence the outcome, and a multi-start approach is strongly recommended to distinguish the global optimum of the function from potential local optima.

Metaheuristic strategies may bring relief for these issues. Metaheuristic strategies are stochastic inverse methods that explore the search space to find (near-)optimal solutions - in this case the hygric material properties - of an optimisation problem. In general, metaheuristics are computationally more demanding than a single run with a gradient-based technique; though their higher flexibility and stochastic sampling strategy might be beneficial in many optimisation problems, especially when dealing with non-linear problems. Indeed, metaheuristic strategies all pursue reaching a global optimum while avoiding getting stuck in a local suboptimum. Three main types of metaheuristics can be distinguished: metaheuristics based on evolutionarycomputation (EC), swarm-intelligence (SI) and physics-based algorithms. The most popular metaheuristic based on evolutionary computation is definitely the Genetic Algorithm [20,21], which has already been applied by Koči et al. [22] for the determination of the water vapour diffusion parameters of building materials. Another example is the Covariance Matrix Adaptation Evolution Strategy [23], which has been applied by Rouchier et al. [24] for the identification of the hygrothermal properties of building envelope materials. Over the past few years, however, a multitude of new 
Postprint: Vereecken E, Roels S, Janssen H, 2018. Inverse hygric property determination based on dynamic measurements and swarm-intelligence optimisers, Building and Environment 131: 184-196.

https://doi.org/10.1016/j.buildenv.2017.12.030

swarm-intelligence strategies have been developed. These strategies are inspired by the collective behaviour and intelligence of swarms. A single swarm individual has only limited capabilities to reach an optimal solution. By interaction with other swarm individuals, and thus driven by both their own and neighbours' best performances, however, they show a good global behaviour and are provided with an enlarged capability reaching an optimal solution. Examples of algorithms that make use of swarm intelligence are Particle Swarm Optimisation [25,26,27], Artificial Bee Colony [28,29], Ant Colony Optimisation [30], Firefly algorithm [31,32], Bat Algorithm [32] and Cuckoo Search $[33,34]$. Compared to these evolutionary-computation and swarmintelligence algorithms, physics-based strategies are less known by researchers. A closer look does however reveal that this third type of metaheuristic is widely represented as well [35]. Two examples of this type are the Gravitational Search Algorithm [36,37] and the Big Bang-Big Crunch Algorithm [38]. Finally, a rising trend of hybrid metaheuristics, aiming an improved performance by combining components from different metaheuristics, can be observed $[39,40]$.

This paper studies the applicability of two swarm-intelligence algorithms, i.e. the Particle Swarm Optimiser (PSO) and the Grey Wolf Optimiser (GWO), for the processing and interpretation of dynamic hygric measurements in order to characterise the hygric properties of porous building materials in a shorter time span. The PSO, originally developed by Eberhart and Kennedy [25], is one of the most popular SI algorithms [41,42]. On the other hand, the GWO, developed by Mirjalili et al. [43], is a more recent metaheuristic technique. Notwithstanding its rather recent development, however, this optimiser has already been applied in many science disciplines (a.o. [44- 
Postprint: Vereecken E, Roels S, Janssen H, 2018. Inverse hygric property determination based on dynamic measurements and swarm-intelligence optimisers, Building and Environment 131: 184-196.

https://doi.org/10.1016/j.buildenv.2017.12.030 48]) and is often shown to outperform other metaheuristic strategies (a.o. $[48,49,50]$ ). In the building physics field, however, the potential of both SI algorithms has, to the best of the authors' knowledge, not been studied yet. Additional research is needed to assess the applicability and the potential added value of these optimisation algorithms in the building physics field. Indeed, given the impossibility of a general-purpose and universal optimisation strategy as implied by the No Free Lunch (NFL) theorem $[51,52]$, the findings obtained in previous studies in other fields may not directly be extrapolated.

The paper is organised as follows. In Section 2, first the general principles of parameter estimation are reiterated. Next, Section 3 explains the working mechanism of the Particle Swarm and Grey Wolf Optimisers. In Section 4, both optimisers are applied to a numerical benchmark, i.e. a dynamic sorption experiment, and this with the aim of estimating the hygric properties. A profile likelihood analysis is applied to inspect the identifiability and to appraise the confidence of the parameter estimates. Additionally, the PSO's and GWO's performance are compared to the performance of a Genetic Algorithm (GA) and the Covariance Matrix Adaptation Evolution Strategy (CMA-ES). Finally, the crucial findings are discussed and the main conclusions are drawn.

\section{Material characterisation based on parameter estimation}

In the last decades, parameter estimation strategies for material characterisation have gained popularity in soil science $[53,54,55]$ and other disciplines [56,57]. Also in building physics parameter estimation techniques pop up, though more slowly and often focused on the building component and building scale (a.o. $[58,59,60])$; for hygric 
Postprint: Vereecken E, Roels S, Janssen H, 2018. Inverse hygric property determination based on dynamic measurements and swarm-intelligence optimisers, Building and Environment 131: 184-196.

https://doi.org/10.1016/j.buildenv.2017.12.030

material characterisation the examples are more scarce [22,24,61]. Figure 1 shows the global workflow of a parameter estimation process applied to material characterisation based on dynamic measurements. An experimental and a numerical part can be distinguished. In the experimental part (Part A), a system - in this case the sample - is exposed to specific boundary conditions, resulting in a specific (set of) test result(s). Due to the presence of e.g. measurement noise on the measurement equipment, the observed outcome can deviate from the actual outcome caused by the implemented boundary conditions. Similarly, measurement noise due to e.g. sensor inaccuracies makes that the observed input conditions may differ from the actual experimental boundary conditions. In the parameter estimation part (Part B), the observed input conditions, thus the experimental boundary conditions including measurement noise, is imposed to a hygric model incorporated in a parameter estimation model. Deviations between this hygric model and practice might result in system noise. In the parameter estimation model, the simulated output of the hygric model is compared to the measured output by use of an objective or fitness function. To find the near-optimal solution, the metaheuristic algorithm explores the search space, starting from an initial guess for the candidate solutions. In this search the information achieved in the prior iterations is used to suggest other candidate solutions that minimise the residuals between measured and simulated results (or maximise the likelihood), as described by the objective function. In the current study, this optimisation is performed by the Particle Swarm Optimiser (PSO) and the Grey Wolf Optimiser (GWO), which are explained in the next section. Additionally, a comparison is made with the Genetic Algorithm (GA) and the Covariance Matrix Adaptation Evolution Strategy (CMA-ES). 
Postprint: Vereecken E, Roels S, Janssen H, 2018. Inverse hygric property determination based on dynamic measurements and swarm-intelligence optimisers, Building and Environment 131: 184-196.

https://doi.org/10.1016/j.buildenv.2017.12.030

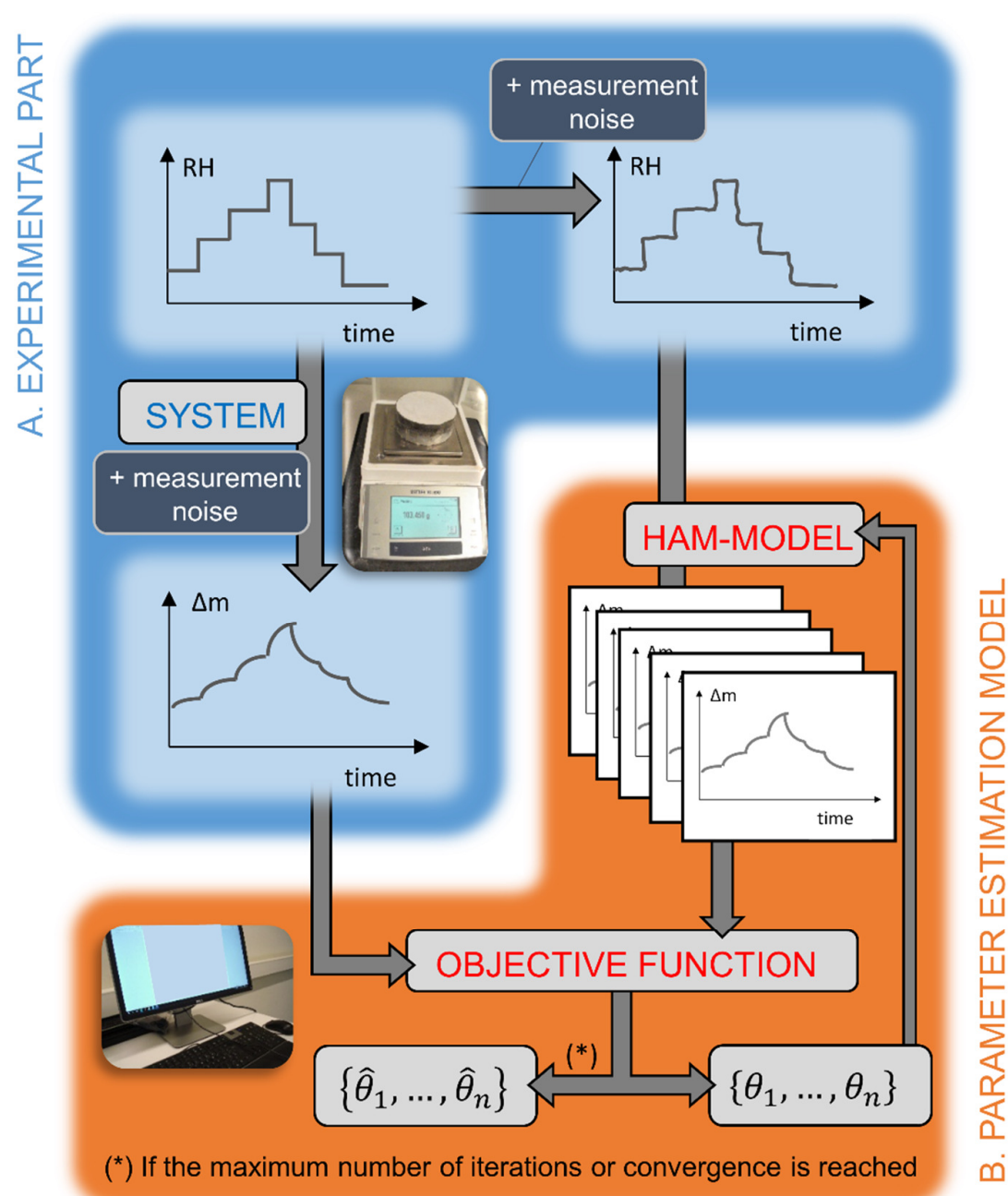

Figure 1. Parameter estimation workflow. 
Postprint: Vereecken E, Roels S, Janssen H, 2018. Inverse hygric property determination based on dynamic measurements and swarm-intelligence optimisers, Building and Environment 131: 184-196.

https://doi.org/10.1016/j.buildenv.2017.12.030

\section{Description of the swarm-intelligence algorithms}

\subsection{Particle Swarm Optimiser}

The Particle Swarm Optimiser (PSO) [41] is an optimisation algorithm inspired by the social behaviour and the information circulation observed in bird flocks or fish schools. The optimisation algorithm has two main features: (1) the search particles are characterised by a position as well as by a velocity and (2) the search particles' movement is influenced by both their own memory and the best position found by other particles.

The mathematical translation of the information transfer and the social behaviour observed in bird flocks and fish schools is described in what follows. For a more extended description of the different parameters and further developments of PSO, the reader is referred to $[26,27]$.

The position of the $i^{\text {th }}$ particle of the swarm is, for a $n_{p}$-dimensional search space, given by the vector:

$$
\boldsymbol{x}_{\boldsymbol{i}}=\left[\begin{array}{llll}
x_{i, 1} & x_{i, 2} & \ldots & x_{i, n_{p}}
\end{array}\right]
$$

The population of $\mathrm{N}$ particles constitutes the swarm, which is indicated by the matrix:

$$
X=\left\{x_{1}, x_{2}, \ldots, x_{N}\right\}
$$

The swarm particles define trajectories in the search space based on the following equation of motion:

$$
x_{i}(t+1)=x_{i}(t)+v_{i}(t+1)
$$


Postprint: Vereecken E, Roels S, Janssen H, 2018. Inverse hygric property determination based on dynamic measurements and swarm-intelligence optimisers, Building and Environment 131: 184-196.

https://doi.org/10.1016/j.buildenv.2017.12.030

with $t$ the current iteration step and $\boldsymbol{v}_{\boldsymbol{i}}$ the $n_{p}$-dimensional velocity vector of the $\mathrm{i}^{\text {th }}$ particle. This velocity vector describes - along the $n_{p}$ dimensions - the way the particle moves across the search space and is given by:

$$
\boldsymbol{v}_{\boldsymbol{i}}(t+1)=\underbrace{w \cdot \boldsymbol{v}_{\boldsymbol{i}}(t)}_{(\mathbf{1})}+\underbrace{c_{1} \cdot\left(\boldsymbol{p}_{\boldsymbol{i}}-\boldsymbol{x}_{\boldsymbol{i}}(t)\right) \cdot \boldsymbol{R}_{\mathbf{1}}}_{(2)}+\underbrace{c_{2} \cdot\left(\boldsymbol{g}-\boldsymbol{x}_{\boldsymbol{i}}(t)\right) \cdot \boldsymbol{R}_{\mathbf{2}}}_{(3)}
$$

Three terms can be distinguished in Eq.(4):

(1) the inertia or momentum, which prevents a too drastic change in direction by taking into account the previous flow direction;

(2) the cognitive component, which reckons the particle's tendency to return to its own previously found best position. Herein, $\boldsymbol{p}_{\boldsymbol{i}}$ is the particle's 'personal best' position so far. An acceleration constant $c_{1}$, often called the cognitive or personal learning coefficient, sets the particle's step size towards its personal best position. A stochastic behaviour is implemented by $\boldsymbol{R}_{\boldsymbol{1}}$, which is a $n_{p} \times n_{p}$ diagonal matrix containing random values in $[0,1]$.

(3) the social component, which reckons the particle's tendency to move towards the best position of the whole swarm. Herein, $\boldsymbol{g}$ is the 'global best' position obtained by the swarm. The acceleration factor $c_{2}$, often called the social or global learning coefficient, sets the particle's step size towards the global best position. As with the cognitive component, a stochastic behaviour is implemented by the diagonal $\mathrm{n}_{\mathrm{p}}$ dimensional matrix $\boldsymbol{R}_{\mathbf{2}}$, which contains randomly generated numbers in the range $[0,1]$. 
Postprint: Vereecken E, Roels S, Janssen H, 2018. Inverse hygric property determination based on dynamic measurements and swarm-intelligence optimisers, Building and Environment 131: 184-196.

https://doi.org/10.1016/j.buildenv.2017.12.030

\subsection{Grey Wolf Optimiser}

The Grey Wolf Optimiser (GWO) algorithm [43] is a nature-inspired optimisation algorithm that mimics the leadership hierarchy and hunting behaviour of grey wolves

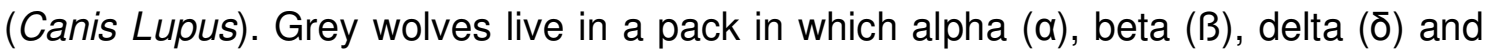
omega $(\omega)$ wolves can be distinguished. An $\alpha$-wolf, positioned on top of the pack, decides on the hunting mechanism and other vital activities. The other wolves should follow the orders of the a-wolf. Grey wolves show an interesting group hunting behaviour. The main parts in the hunting process are (1) tracking, chasing, and approaching the prey, (2) encircling, pursuing, and harassing the prey until it stops moving and (3) attacking the prey.

The social hierarchy and the hunting behaviour of the grey wolves as translated in a mathematical model by [43] are described in what follows.

\section{- Social hierarchy}

In the GWO, the three fittest solutions - indicated as $\alpha$, $\beta$ - and $\delta$-wolves - guide the optimisation process. The other candidate solutions ( $\omega$-wolves) follow these three fittest wolves. At the start of the optimisation process, a random population of candidate solutions - describing the positions of the wolves - is generated.

\section{- Hunting behaviour}

The grey wolves' group hunting behaviour fits perfectly in the principle of swarm intelligence. At the start of the grey wolves' hunting, the wolves (search agents) try to trace a prey. When they have traced a prey, they encircle the prey and finally attack the prey. In the GWO algorithm, tracing the prey corresponds to the exploration phase, 
Postprint: Vereecken E, Roels S, Janssen H, 2018. Inverse hygric property determination based on dynamic measurements and swarm-intelligence optimisers, Building and Environment 131: 184-196.

https://doi.org/10.1016/j.buildenv.2017.12.030

whereas approaching and attacking the prey is modelled as the exploitation phase. The search agents that constitute the pack are each defined by a position in the search space. The motion of the pack, is mathematically described as (given per search agent x) [43]:

$$
\begin{gathered}
\boldsymbol{x}(t+1)=\boldsymbol{x}_{\boldsymbol{p}}(t)-\boldsymbol{A} \cdot \boldsymbol{D} \\
\boldsymbol{D}=\left|\boldsymbol{C} \cdot \boldsymbol{x}_{\boldsymbol{p}}(t)-\boldsymbol{x}(t)\right|
\end{gathered}
$$

with $t$ the current iteration step, $\boldsymbol{x}_{\boldsymbol{p}}$ and $\boldsymbol{x} n_{p}$-dimensional vectors (for a $n_{p}$-dimensional search space) indicating the position of the prey and the grey wolf concerned, respectively, and $\boldsymbol{A}$ and $\boldsymbol{C} \mathrm{n}_{\mathrm{p}} \times \mathrm{n}_{\mathrm{p}}$ diagonal coefficient matrices given by

$$
\begin{gathered}
A=2 \cdot a \cdot r_{1}-a \\
C=2 \cdot r_{2}
\end{gathered}
$$

with $a$ a $n_{p} \times n_{p}$ diagonal matrix with values that linearly decreases from 2 to 0 over the course of the iterations and $\boldsymbol{r}_{1}$ and $\boldsymbol{r}_{2} \mathrm{n}_{\mathrm{p}} \times \mathrm{n}_{\mathrm{p}}$ diagonal matrices with random values in the interval $[0,1]$. By modifying the values in $\boldsymbol{A}$ and $\boldsymbol{C}$, the search agents can reach different positions around the prey.

In an optimisation problem the exact location of the optimum (prey) is, however, unknown. Thus, an assumption for the vector $\boldsymbol{x}_{\boldsymbol{p}}$ in Eq.(5) should be made. In the GWO the $\alpha, \beta$ - and $\delta$-wolves are assumed to have better knowledge on the potential location of the prey. Therefore, the position of these three wolves is used to update the position of the entire pack (with inclusion of the $\omega$-wolves), as described by the following equations (given per search agent $\boldsymbol{x}$ ): 
Postprint: Vereecken E, Roels S, Janssen H, 2018. Inverse hygric property determination based on dynamic measurements and swarm-intelligence optimisers, Building and Environment 131: 184-196.

https://doi.org/10.1016/j.buildenv.2017.12.030

$$
D_{\alpha}=\left|C_{1} \cdot x_{\alpha}-x\right|, D_{\beta}=\left|C_{2} \cdot x_{\beta}-x\right|, D_{\delta}=\left|C_{3} \cdot x_{\delta}-x\right|
$$

with $\boldsymbol{x}_{\alpha / \beta / \delta} \mathrm{n}_{\mathrm{p}}$-dimensional vectors indicating the position of the three best search agents, $\boldsymbol{C}_{\mathbf{1 / 2} / \mathbf{3}}$ random $\mathrm{n}_{\mathrm{p}}$-dimensional diagonal matrixes and $\boldsymbol{x}$ a $\mathrm{n}_{\mathrm{p}}$-dimensional vector that specifies the current position of the investigated search agent. The matrices $D_{\alpha / \beta / \delta}$ define the span between the current position of a search agent and the position of the $\alpha / ß / \delta$-wolf. This span is used to determine the updated position of each of the search agents by:

$$
\begin{gathered}
x_{1}=x_{\alpha}-A_{1} \cdot D_{\alpha}, x_{2}=x_{\beta}-A_{2} \cdot D_{\beta}, x_{3}=x_{\delta}-A_{3} \cdot D_{\delta} \\
x(t+1)=\frac{x_{1}+x_{2}+x_{3}}{3}
\end{gathered}
$$

with $t$ the current iteration step and $\boldsymbol{A}_{1 / 2 / 3} n_{p a r} \times n_{p a r}$ diagonal coefficient matrices (Eq.(7)). In this way, over the course of iterations, $\alpha, \beta$ - and $\delta$-wolves assess the probable position of the prey. Next, the distance between the candidate solutions (updated position of the wolves) and the prey is computed by use of the objective function, on the basis of which new $\alpha, \beta$ - and $\delta$-wolves are nominated. The matrices $\boldsymbol{A}_{(1 / 2 / 3)}$ in Eq.(10) decide on the exploitation and exploration strength. The elements in $\boldsymbol{A}$ can take a value in the range $[-2 \mathrm{a}, 2 \mathrm{a}]$, with the parameter ' $a$ ' decreasing from 2 to 0 over the course of the iterations. An absolute value larger than 1 for the elements in $\boldsymbol{A}$ makes that the search agents explore the search space; they are forced to diverge from the prey and, thus, to search globally. If the absolute value of the elements in $\boldsymbol{A}$ is smaller than 1, exploitation takes place; the search agents are forced to attack the prey. Due to the implementation of the parameter ' $a$ ', which decreases linearly over 
Postprint: Vereecken E, Roels S, Janssen H, 2018. Inverse hygric property determination based on dynamic measurements and swarm-intelligence optimisers, Building and Environment 131: 184-196.

https://doi.org/10.1016/j.buildenv.2017.12.030

the course of iterations, the first half of the optimisation process focuses on exploration, while as the process progresses the focus gradually shifts to exploitation. This focus on exploitation can, however, be discouraged by the matrix $\boldsymbol{C}$ (Eq.(8)). The values of this matrix can take random values in the range $[0,2]$, and this at all times of the optimisation process. In this way, also in the later iteration steps exploration is encouraged and local optima entrapment is avoided.

\section{Application}

\subsection{Benchmark description}

The Particle Swarm Optimiser and the Grey Wolf Optimiser are applied to determine the hygric properties, i.e. the vapour resistance factor and the sorption isotherm, of a calcium silicate insulation sample, and this based on the output of a dynamic vapour sorption experiment. To get a clear view on the deviation from the actual material properties searched for, a fictitious test case is generated. After all, material properties obtained based on real (steady-state) measurements could be biased $[7,62,63]$; which in its turn might hinder a fair assessment of the applicability of the optimisation methodology.

The calcium silicate insulation's actual sorption isotherm and vapour resistance factor as a function of relative humidity are shown in Figure 2. Here, the sorption isotherm is described by a unimodal Van Genuchten function, relating the moisture content $w$ $\left(\mathrm{kg} / \mathrm{m}^{3}\right)$ to the relative humidity $\Phi(-)$ :

$$
w(\phi)=w_{\text {sat }} \cdot\left(1+(k \cdot \ln (\phi))^{n}\right)^{-m}
$$


Postprint: Vereecken E, Roels S, Janssen H, 2018. Inverse hygric property determination based on dynamic measurements and swarm-intelligence optimisers, Building and Environment 131: 184-196.

https://doi.org/10.1016/j.buildenv.2017.12.030

where $w_{s a t}, k, n$ and $m$ are fitting parameters. The vapour resistance factor $\mu(-)$ in function of relative humidity $\Phi(-)$ is described by:

$$
\mu(\phi)=\frac{1}{a+b \cdot \exp (c \cdot \phi)}
$$

where $a, b$ and $c$ are fitting parameters. To generate the synthetic data the fitting parameters are set to: $w_{\text {sat }},=811.14, k=-2.6594 \times 10^{3}, n=1.761$ and $m=(n-1) / n=$ $0.4321, a=0.174, b=2.806 \times 10^{-4}$ and $c=7.229$. The synthetic data created in this way agree with the calcium silicate insulation's hygric properties obtained by Roels et al. [65]. 
(a)

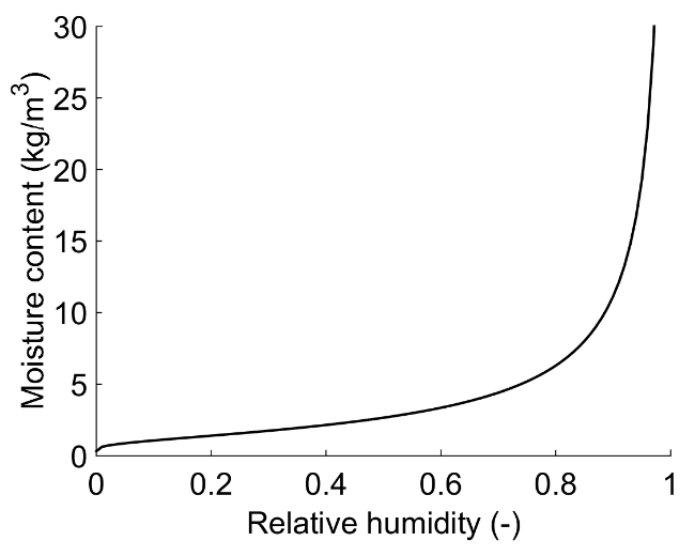

(b)

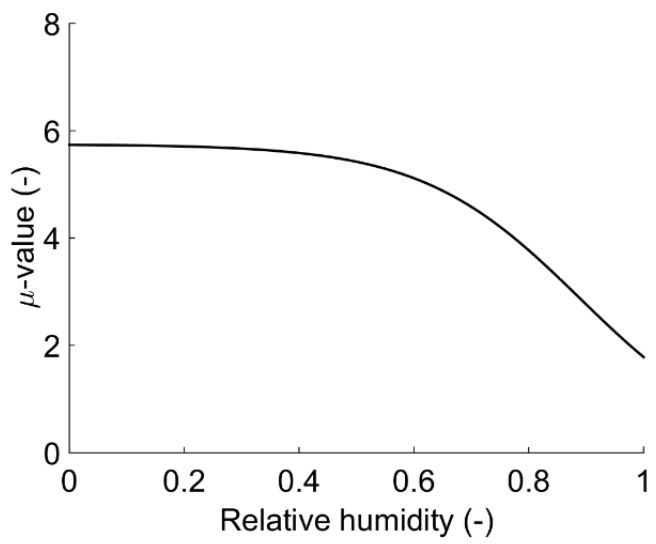

Figure 2. Hygric properties of the calcium silicate sample (target properties) based on [65]: (a) sorption isotherm, (b) vapour diffusion resistance.

In the fictitious test case, a cylindrical sample with a diameter of $8 \mathrm{~cm}$ and a height of $4 \mathrm{~cm}$ is exposed to dynamic relative humidity conditions. All sides except for the top of the sample are covered with a vapour tight foil. In this way, one-dimensional moisture transport is ensured. The mass transfer coefficient is set to $3 \cdot 10^{-8} \mathrm{~s} / \mathrm{m}+\mathcal{N}\left(\mu, \sigma^{2}\right)$ with $\mu=0$ and $\sigma=2 \cdot 10^{-9}$. Before starting the experiment, the calcium silicate is oven-dried at $12 \%$ relative humidity. The temperature in the fictitious experiment is set to $20^{\circ} \mathrm{C}$. The dynamic step function in relative humidity to which the sample is exposed is as follows: $24 \mathrm{~h} 33 \% \mathrm{RH}, 24 \mathrm{~h} 54 \% \mathrm{RH}, 24 \mathrm{~h} 75 \% \mathrm{RH}$ and $24 \mathrm{~h} 85 \% \mathrm{RH}$. The mass change of the sample and the relative humidity at the bottom of the sample are assumed to be the measured output of the experiment. To generate these synthetic measurement outputs, the moisture transfer in the sample is simulated one-dimensionally, by using 
Postprint: Vereecken E, Roels S, Janssen H, 2018. Inverse hygric property determination based on dynamic measurements and swarm-intelligence optimisers, Building and Environment 131: 184-196.

https://doi.org/10.1016/j.buildenv.2017.12.030

a control volume model which solves the partial differential equation for moisture transfer:

$$
\frac{\partial w}{\partial \phi} \frac{\partial \phi}{\partial t}-\nabla\left[\frac{\delta_{\text {air }}}{\mu(\phi)} \nabla \phi p_{\text {sat }}\right]=0
$$

with $w$ the moisture content $\left(\mathrm{kg} / \mathrm{m}^{3}\right), \phi$ the relative humidity [-], $\mu$ the vapour resistance factor (-), $\delta_{\text {air }}$ the air permeability (s) and $p_{\text {sat }}$ the saturated vapour pressure $(\mathrm{Pa})$. The boundary condition at the top of the sample is given by the vapour flux $g_{v}$ :

$$
g_{v}=\beta \cdot\left(p_{v, \text { air }}-p_{v, \text { surf }}\right)
$$

with $\beta$ the moisture transfer coefficient $(\mathrm{s} / \mathrm{m}), p_{v, \text { air }}$ the partial vapour pressure in the air above the sample and $p_{v, \text { surf }}(\mathrm{Pa})$ the partial vapour pressure at the top surface of the sample $(\mathrm{Pa})$. Temporal and spatial discretisation is applied. To reach a converged solution an iterative process is implemented within each time step. The convergence criteria employed in this iterative process is:

$$
\left\|\frac{p_{v}^{t+\Delta t, i t+1}-p_{v}^{t+\Delta t, i t}}{p_{v}^{t+\Delta t, i t}}\right\|<10^{-6}
$$

with $p_{v}$ the vapour pressure in the different control volumes, $t$ the time, $\Delta t$ the time step and it the iteration step in the control volume method. The maximum number of iterations in the control volume method is set to 10 . No heat transfer is simulated as isothermal boundary conditions are assumed in the numerical experiment and latent heat transfer is neglected.

In practice, both the synthetic experimental input conditions and the synthetic measured output will be subjected to measurement noise. In the current study, measurement noise due to the inaccuracy of e.g. the relative humidity sensor is added 
Postprint: Vereecken E, Roels S, Janssen H, 2018. Inverse hygric property determination based on dynamic measurements and swarm-intelligence optimisers, Building and Environment 131: 184-196.

https://doi.org/10.1016/j.buildenv.2017.12.030

to the relative humidity to which the sample is exposed. This is done by adding Gaussian noise $\varepsilon \sim \mathcal{N}\left(\mu, \sigma^{2}\right)$ with the mean $\mu$ of the Gaussian noise term set at 0 and the standard deviation $\sigma$ set to 0.015 . The relative humidity course that is achieved in this way is shown in Figure 3 and will be applied as the input in the estimation part. The measurement noise on the synthetic output is in the current case attributed to e.g. the inaccuracy of the balance and of the $\mathrm{RH}$-sensor positioned at the bottom of the sample. To include this measurements noise, again white Gaussian noise $\varepsilon \sim \mathcal{N}\left(\mu, \sigma^{2}\right)$ is added, with the mean $\mu$ set at 0 and the standard deviation $\sigma$ set to 0.015 for the relative humidity measurements and to 0.01 for the mass measurements. Both outputs, with inclusion of the measurement noise, are shown in Figure 4 and are assumed to be logged with a 10 minutes interval. No system noise is included in the current study.

(a)

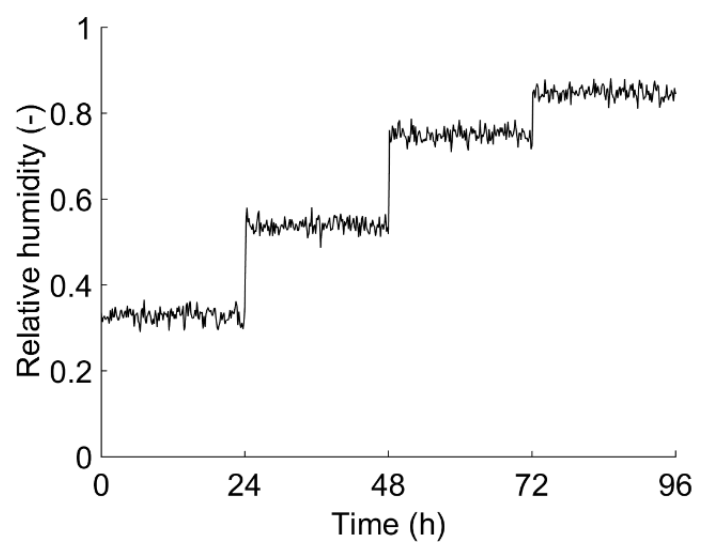

Figure 3. RH-course in the climatic chamber (with inclusion of measurement noise) as assumed in the fictitious experiment. 
(a)

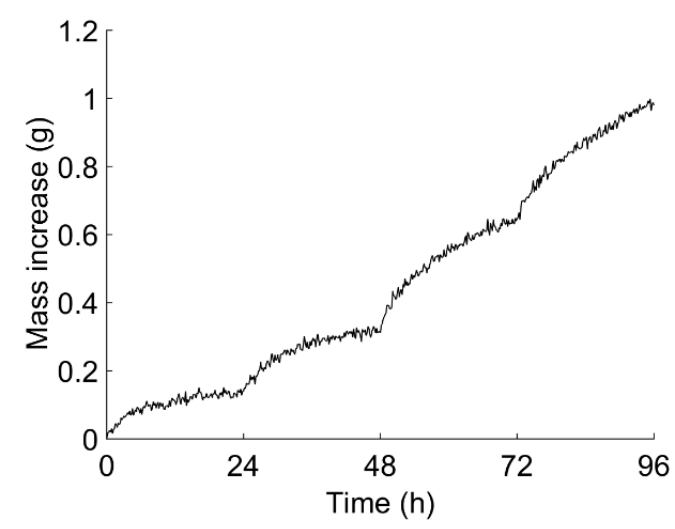

(b)

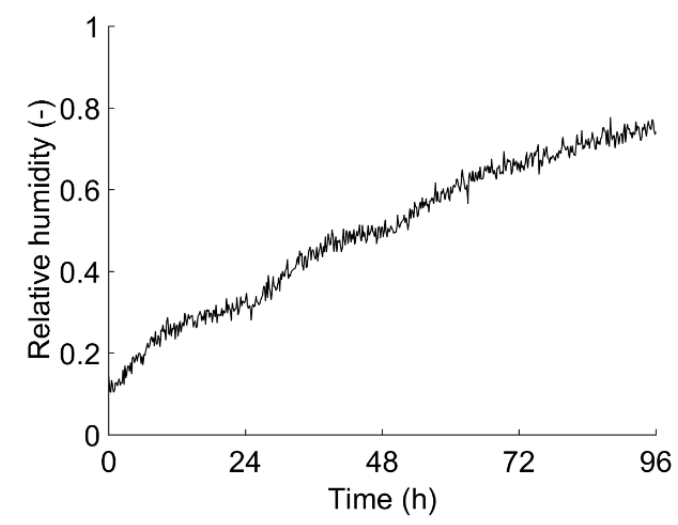

Figure 4. Output of the fictitious experiment: (a) mass increase with inclusion of measurement noise, (b) relative humidity measured at the bottom of the sample with inclusion of measurement noise.

\subsection{Parameter estimation}

To estimate the vapour resistance factor and the sorption isotherm, the parameters $m$ (Eq.(12)) and $a, b$ and $c$ (Eq.(13)) are searched for. The parameter $n$ in Eq.(12) is set to $1 /(1-\mathrm{m})$ and the parameter $w_{\text {sat }}$ in Eq.(12) is set to the saturated moisture content $\left(811.14 \mathrm{~kg} / \mathrm{m}^{3}\right)$. As long as the latter parameter is set to a large enough value, it should be possible to describe the synthetic data based on the Van Genuchten function (Eq.(12)). By setting $w_{\text {sat }}$ to the same value as applied for the generation of the synthetic data, a comparison between the estimated and the target parameter $\mathrm{m}$ remains possible when studying the performance of the optimisation process. Additionally, the moisture content at $54 \%$ relative humidity is assumed to be known $\left(2.9 \mathrm{~kg} / \mathrm{m}^{3}\right)$ and is used to define the parameter $\mathrm{k}$ as a function of the other parameters: 
Postprint: Vereecken E, Roels S, Janssen H, 2018. Inverse hygric property determination based on dynamic measurements and swarm-intelligence optimisers, Building and Environment 131: 184-196.

https://doi.org/10.1016/j.buildenv.2017.12.030

$$
k=\left[\left(\frac{w_{0.54}}{w_{\text {sat }}}\right)^{\frac{-1}{m}}-1\right]^{\frac{1}{n}} \cdot \frac{1}{\ln (0.54)}
$$

Such a single fixed value of the sorption isotherm ensures that the estimation of the moisture capacity $(\partial w / \partial \phi)$, which is used in the partial differential equation for moisture transfer (Eq.(14)), is sufficient to describe the entire sorption isotherm. To limit the extra time required to achieve one point of the sorption isotherm in advance, it is recommended to define the moisture content at a relative humidity close to the relative humidity at which the samples are assumed to be before oven drying is applied. Another option is to replace the Van Genuchten function - which is applied to describe the sorption isotherm - by a function that exists for $\phi$ equal to zero. In this way, $w_{0}=0$ $\mathrm{kg} / \mathrm{m}^{3}$ can be applied as the single fixed value. The estimation of the parameters of a parametric function is in the current study preferred above a direct estimation of physical parameters (e.g. $\mu_{54}, w_{54}, \ldots$ ), such as applied by Rouchier et al. [66], as a direct estimation requires the implementation of a physical relation between the physical parameters searched for (for example $\mu_{54}=\mathrm{a} \cdot \mu_{85}$ with a $>1$ ) or the introduction of a penalty function in the optimisation algorithm to excluded non-physical combinations. Such additional measures in the direct estimation process would complicate the optimisation, especially when dealing with a large number of unknown parameters.

Table 1 gives an overview of the different parameters searched for, together with the upper and lower boundaries of their search space as applied in the parameter estimation. For the diffusion resistance factor, these boundaries are defined in a way that the dry vapour diffusion resistance can range between $\mu=1.25$ and $\mu=20$. 
Postprint: Vereecken E, Roels S, Janssen H, 2018. Inverse hygric property determination based on dynamic measurements and swarm-intelligence optimisers, Building and Environment 131: 184-196.

https://doi.org/10.1016/j.buildenv.2017.12.030

Additionally, physically non-plausible courses showing a rather steep change in vapour diffusion resistance factor in the range below $50 \%$ relative humidity are partially excluded by restricting the boundaries for the parameters $b$ and $c$. For the parameter $m$ which defines the moisture content, a rather broad range is set, ranging from no moisture in the sample at all $\left(\mathrm{w} \approx 0 \mathrm{~kg} / \mathrm{m}^{3}\right)$ till a moisture content around $400 \mathrm{~kg} / \mathrm{m}^{3}$ at $80 \%$ relative humidity. Another parameter that is treated as an unknown is the mass transfer coefficient ß. Finally, also the measurement noise is unknown. If measurement replicates are available, this measurement noise could be estimated on this basis. In the current study, however, the variance of the measurement noise $\left(\sigma_{M}\right.$ and $\left.\sigma_{R H}\right)$ is estimated simultaneously with the hygric properties. For the parameter estimation process all parameters in the search space are log-transformed. For reasons of readability, however, in the figures and in the text only the parameters $b, \sigma_{M}, \sigma_{R H}$ and $\beta$ are shown on a log-scale.

Table 1. Parameters and lower and upper boundaries assumed in the parameter estimation search space

\begin{tabular}{ll}
\hline Variable & Search space \\
\hline$a$ & {$[0.05,0.8]$} \\
$b$ & {$\left[10^{-10}, 10^{-2}\right]$} \\
$c$ & {$[4,30]$} \\
$m$ & {$[0.1,0.8]$} \\
$\sigma_{M}$ & {$\left[10^{-5}, 1\right]$} \\
$\sigma_{R H}$ & {$\left[10^{-5}, 1\right]$} \\
$\beta$ & {$\left[10^{-9}, 10^{-6}\right]$} \\
\hline
\end{tabular}


Postprint: Vereecken E, Roels S, Janssen H, 2018. Inverse hygric property determination based on dynamic measurements and swarm-intelligence optimisers, Building and Environment 131: 184-196.

https://doi.org/10.1016/j.buildenv.2017.12.030

In the PSO algorithm the swarm size is set to 10 . In the GWO algorithm 10 search agents are deployed to explore and exploit the search space. For a seven-dimensional $(\mathrm{N}=7)$ search space those settings are in accordance with the population size $n_{\text {pop }}=$ $4+\operatorname{floor}(3 \cdot \ln (N))$ often suggested for CMA-ES [64]. The other settings in the PSO are as follows (see the description in section 3.1 for the purpose of these parameters): $c_{1}=1.5, c_{2}=2, w=1 \cdot w_{d a m p}=1 \cdot 0.99$, minimum velocity $=-0.1$ and maximum velocity $=0.1$.

In the current study, the moisture transfer in the calcium silicate insulation sample is simulated by the same control volume model as used to generate the measurement data. Hence, system noise is assumed not to occur. The input in the synthetic experiment are the steps in relative humidity with inclusion of measurement noise (Figure 3), the hygric material properties as defined by the position of each search agent and Eq. $(12,13)$ and the mass transfer coefficient $ß$, which is also defined by the position of each search agent. The temperature is assumed to be measured and is set to $20^{\circ} \mathrm{C}$. Next, the input parameters are assessed by use of an objective function, which is in the current study described by the log-likelihood (LL). In the assumption of independent Gaussian model residuals, this log-likelihood can be calculated by:

$$
\begin{gathered}
L L=\frac{-n_{m}}{2} \log (2 \pi)-\sum_{t=1}^{n_{m}} \log \left(\hat{\sigma}_{t}\right)-\frac{1}{2} \sum_{t=1}^{n_{m}}\left(\frac{\tilde{y}_{t}-y_{t}}{\hat{\sigma}_{t}}\right)^{2}+\frac{-n_{R H}}{2} \log (2 \pi) \\
-\sum_{t=1}^{n_{R H}} \log \left(\hat{\sigma}_{t}\right)-\frac{1}{2} \sum_{t=1}^{n_{R H}}\left(\frac{\tilde{y}_{t}-y_{t}}{\hat{\sigma}_{t}}\right)^{2}
\end{gathered}
$$

with $n_{m / R H}$ the number of measurement points of the mass increase of the sample and of the relative humidity at the bottom of the sample, $\hat{\sigma}$ the standard deviations of the 
Postprint: Vereecken E, Roels S, Janssen H, 2018. Inverse hygric property determination based on dynamic measurements and swarm-intelligence optimisers, Building and Environment 131: 184-196.

https://doi.org/10.1016/j.buildenv.2017.12.030 measurement error of the observations, $\tilde{y}$ and $y$ the mass increase in the virtual experimental data set and simulated based on the candidate solutions, respectively. Parameter sets with a maximum log-likelihood are pursued by the optimisation algorithm. The way the standard deviations of the noise enter in the log-likelihood function, i.e. in both the denominators of the least squares and in the additional summation terms for the noise in which the standard deviation has an opposite effect on the likelihood, makes that the estimation of the noise can be included in the parameter estimation process. If only the least square term would be taken into account, the log-likelihood would become large for a large standard deviation of the noise, which can complicate the optimisation process. No regularisation is applied in order to avoid a too subjective steering of the candidate solutions. The maximum number of iterations in the GWO algorithm is set to 400 . The parameter estimation is executed on an Intel® Xeon® CPU (E5-1620 v2 @3.70 GHz), 16GB RAM, Windows 7 system using Matlab 2016a.

\subsection{Identifiability analysis}

In the current study the profile likelihood approach developed by Raue et al. [67] is applied to assess the uniqueness of the parameters and how well they can be estimated. This approach enables a simultaneous investigation of the structural and practical identifiability, where structural identifiability indicates if a unique parameterisation is theoretically possible and practical identifiability describes if such a unique parameterisation can be obtained based on the available measurement data. In this way, structural identifiability is an intrinsic property of the model whereas 
Postprint: Vereecken E, Roels S, Janssen H, 2018. Inverse hygric property determination based on dynamic measurements and swarm-intelligence optimisers, Building and Environment 131: 184-196.

https://doi.org/10.1016/j.buildenv.2017.12.030 practical identifiability is related to the amount and quality of the experimental data. The profile likelihood PL function of a parameter $\theta_{i}$ as presented by Raue et al. [67] is given by:

$$
P L\left(\theta_{i}\right)=\max _{\theta_{j \neq i}} L\left(\theta_{j}\right)
$$

with $L$ the likelihood and $\theta_{j}$ the other parameters searched for in the estimation process. Hence, the profile likelihood function for a parameter $\theta_{i}$ can be achieved by reoptimising the parameters $\theta_{j \neq i}$ for fixed values of $\theta_{i}$ within an interval in the search space. For reasons of numerical stability it is often favourable to work with the loglikelihood. In the current study, the log-likelihood is determined based on Eq.(18).

A profile likelihood-based confidence region is defined by [67]:

$$
\left\{\theta_{i} \mid-2 \log \left(P L\left(\theta_{i}\right)\right) \leq-2 \log (\hat{L})+\Delta_{\alpha}\right\}
$$

with $\Delta_{\alpha}$ the $\alpha^{\text {th }}$ quantile of the $\chi_{d f}^{2}$-distribution with the degrees of freedom $d f$ equal to 1 (pointwise intervals) or to the number of parameters (simultaneous intervals) and $\hat{L}$ the maximum likelihood estimate. A confidence interval is indicated by the border of the confidence region. A finite confidence interval indicates practical identifiability. A semi-finite interval indicates practical non-identifiability. If the confidence interval is infinite at both sides, the parameter is also structurally non-identifiable.

\section{Results}

\subsection{Parameter estimation}

Figure 5 shows the outcomes obtained in 100 independent parameter estimations with the PSO and GWO, after performing 400 iterations. The results are shown based on 
Postprint: Vereecken E, Roels S, Janssen H, 2018. Inverse hygric property determination based on dynamic measurements and swarm-intelligence optimisers, Building and Environment 131: 184-196.

https://doi.org/10.1016/j.buildenv.2017.12.030

box plots. The median is indicated by the central mark in these box plots. Additionally, the $25^{\text {th }}$ and $75^{\text {th }}$ percentile are indicated by the edges of the box plots. The most extreme outcomes not considered as an outlier and the outliers are shown by the whiskers and the plus signs, respectively. For the parameters $a, m, \sigma_{M}$ and $\sigma_{R H}$ the median is in close agreement with the target values. For the parameters $b, c$ and $B$ the median deviates (slightly) from the target values and a larger spread on the results is found (for $\beta$ the mean of the target value is used). The PSO is found to have more difficulties estimating the target value for $b$ and $c$; though, also based on the GWO a rather large spread for $b$ is obtained.

The parameters in the parametric function are, however, not an end in itself. Therefore, Figure 6 shows the results for some physical properties $\left(\mu_{33}, \mu_{75}, \mu_{85}, w_{33}, w_{75}\right.$ and $\left.w_{85}\right)$ as obtained based on the estimated parametric functions. In general, a good agreement with the target values is found. However, mainly for the vapour diffusion resistance factors some outliers are detected. This indicates that a parameter estimation based on a single run is not recommended. The largest spread is found for $\mu_{85}$ and can be attributed to a smaller amount of synthetic measurement data in the higher relative humidity range. 
Postprint: Vereecken E, Roels S, Janssen H, 2018. Inverse hygric property determination based on dynamic measurements and swarm-intelligence optimisers, Building and Environment 131: 184-196.

https://doi.org/10.1016/j.buildenv.2017.12.030
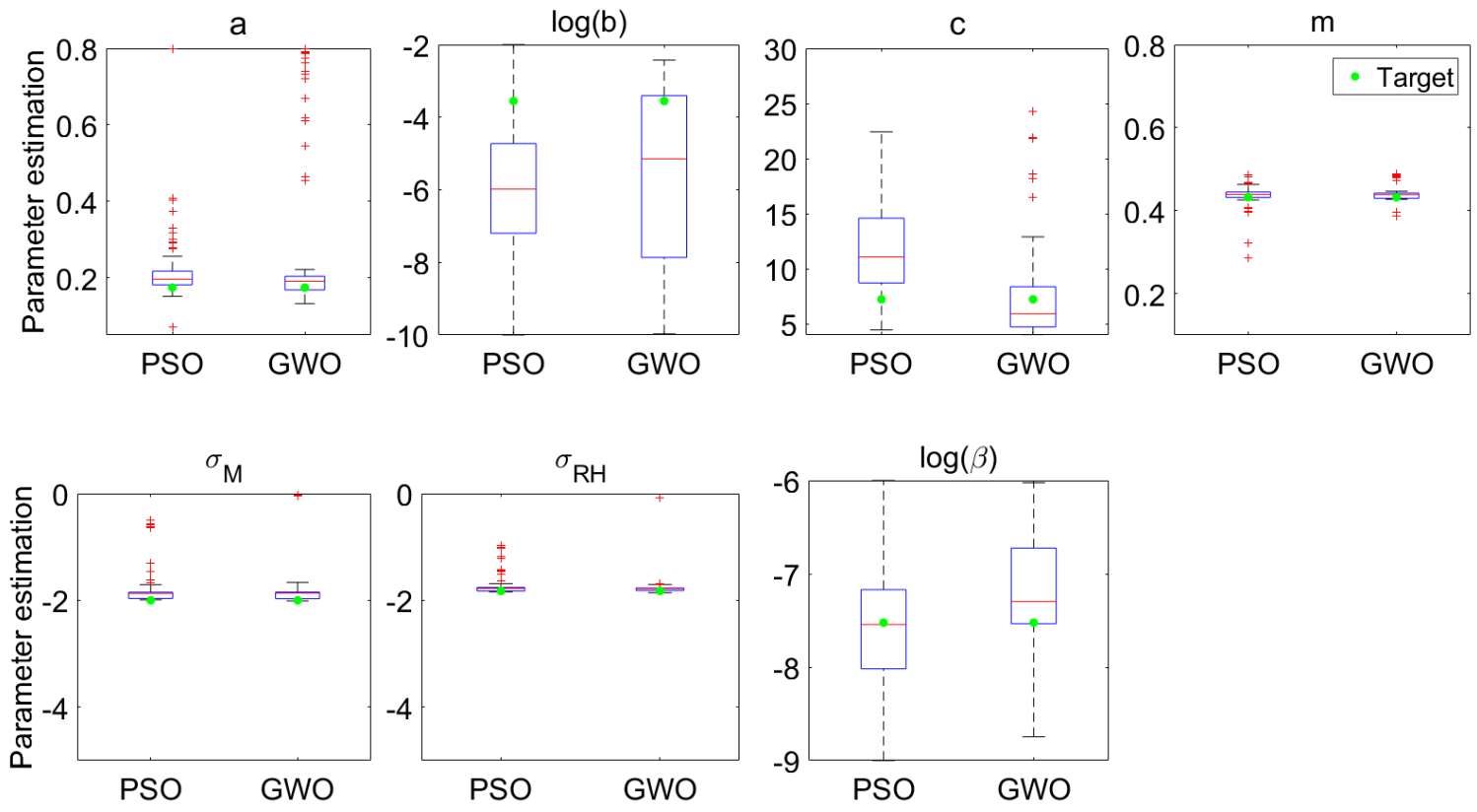

Figure 5. Parameter estimation (after 400 iterations) obtained with the PSO and GWO for 100 independent runs. 
Postprint: Vereecken E, Roels S, Janssen H, 2018. Inverse hygric property determination based on dynamic measurements and swarm-intelligence optimisers, Building and Environment 131: 184-196.

https://doi.org/10.1016/j.buildenv.2017.12.030
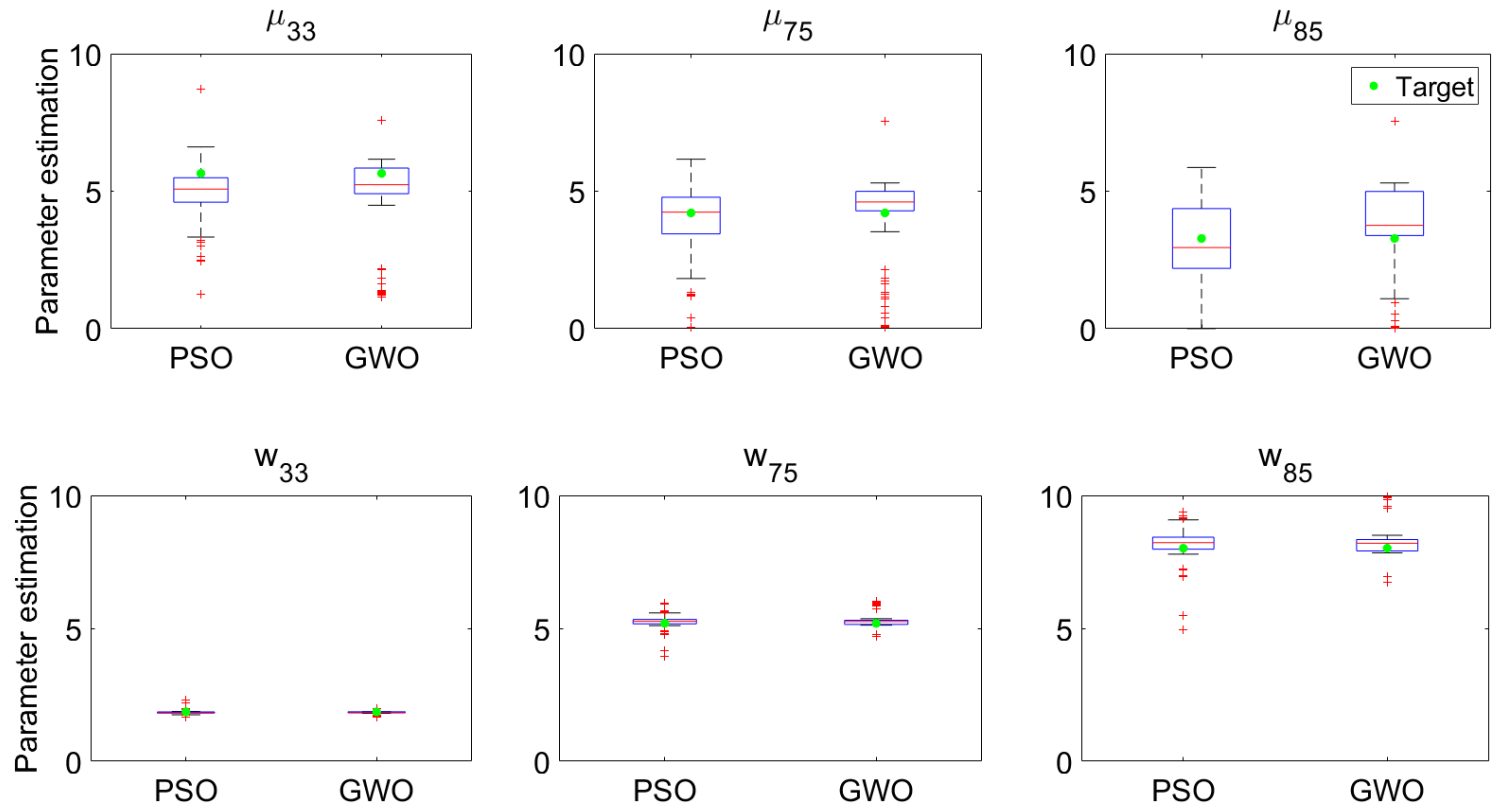

Figure 6. Estimation of the physical material properties (after 400 iterations) obtained with the PSO and GWO for 100 independent runs.

Figure 7 compares the hygric properties over the full relative humidity range as estimated by use of the PSO and GWO to the target properties. Here the 100 independent runs are randomly divided in 10 sets of 10 runs and for each set the best run (resulting in the highest likelihood) is selected. The 10 selected runs yield an acceptable till excellent outcome. For the best run, the assumption of Gaussian noise is checked based on QQ-plots (Figure 8) and is found to be justified. 
(a)

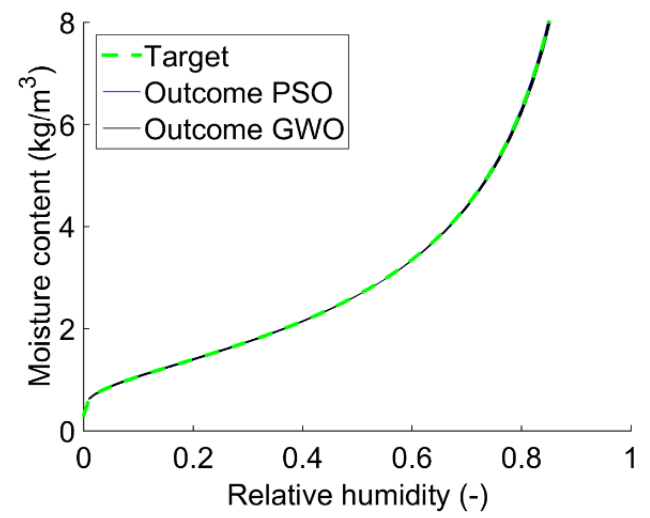

(b)

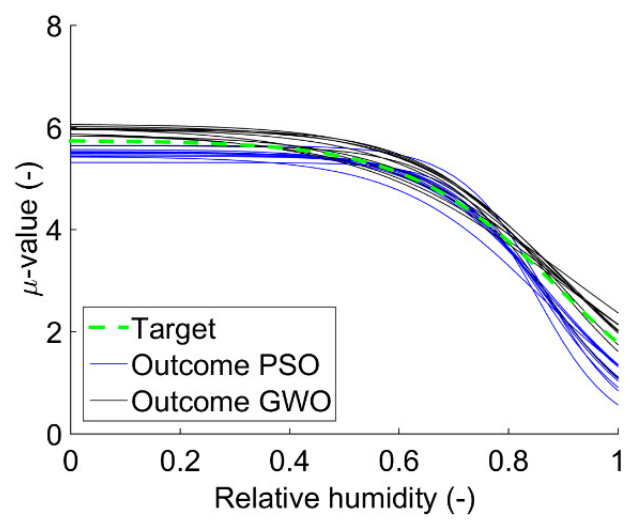

Figure 7. Comparison between target and estimated properties: (a) sorption isotherm and (b) vapour diffusion resistance.

(a)

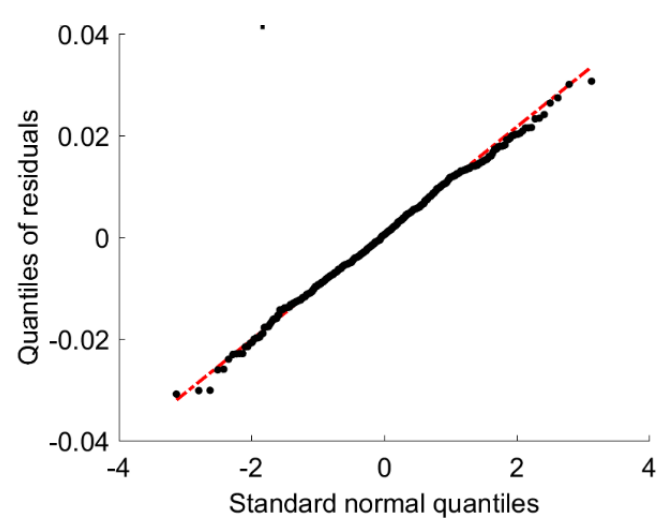

(b)

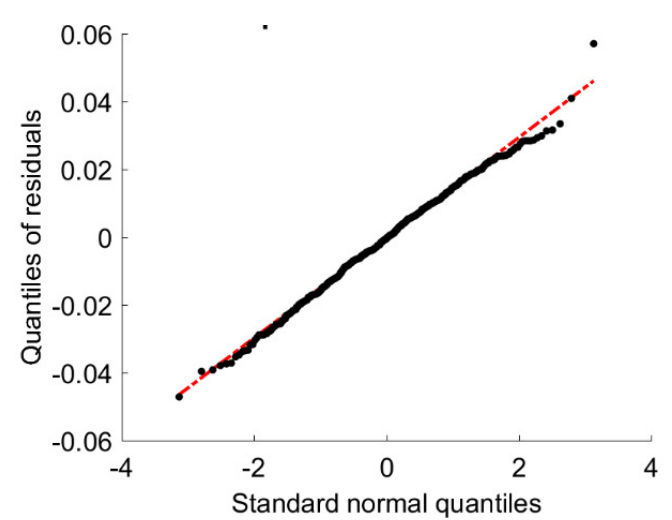

Figure 8. QQ-plot for the best run obtained with GWO: (a) mass increase, (b) relative humidity at the bottom of the sample. 
Postprint: Vereecken E, Roels S, Janssen H, 2018. Inverse hygric property determination based on dynamic measurements and swarm-intelligence optimisers, Building and Environment 131: 184-196.

https://doi.org/10.1016/j.buildenv.2017.12.030

\subsection{Identifiability analysis}

As in practice the target values are unknown, a direct reliability check as performed in the previous section is not possible. Therefore, in the current section a profile likelihood analysis is applied. Such an analysis allows to inspect the identifiability of the parameters. Additionally, a confidence interval can be indicated based on this method. To get a first (rough) indication of the identifiability, the candidate solutions analysed during the estimation process together with their corresponding likelihood are plotted (blue and black dots in Figure 9 for PSO and GWO, respectively). Here the likelihood is plotted as the negative of two times the log-likelihood. The lower the plotted value the higher the likelihood and, thus, the better the agreement between the experimental and the model output. For the parameters $a, m, \sigma_{M}$ and $\sigma_{R H}$, the envelope of the candidate solutions roughly indicates a rather narrow parabolic shape (care should be taken with the $\mathrm{x}$-axis as for some of the parameters only a part of the search space is given). Recall that these parameters also showed a small spread based on the box plots in Figure 5. For the other parameters $(b, c$ and $B)$ the parabolic shape is wider or less pronounced.

In a next step, a profile likelihood analysis is performed taken into account the width of the envelope of the candidate solutions evaluated in the original parameter estimation process. Based on this information, for each parameter a range is defined in which a re-optimisation is performed for fixed parameters as indicated by Eq.(18). For example, to obtain the profile likelihood curve of parameter a 20 additional optimisation runs are performed, each of them for a fixed value of the parameter a in the range between 0.15 and 0.20 . For the non-fixed parameters, the search space as defined in Table 1 is 
Postprint: Vereecken E, Roels S, Janssen H, 2018. Inverse hygric property determination based on dynamic measurements and swarm-intelligence optimisers, Building and Environment 131: 184-196.

https://doi.org/10.1016/j.buildenv.2017.12.030

maintained. The PSO is used for the re-optimisations. Because the forced fixed parameters might induce numerical difficulties, the swarm size is increased to 20 and the maximum number of iterations to 1000 . For some optimisation runs convergence was still not reached after 1000 iterations; in that case additional runs - starting from another initial position in the search space - were performed.

For all the parameters the profile likelihood curves show a clear parabola. A broad parabola indicates a large uncertainty due to e.g. measurement noise, whereas a narrow parabola indicates that the parameter can be estimated with high confidence. By performing the re-optimisation runs, a parameter combination resulting in a slightly higher likelihood is detected. Based on this maximum likelihood estimate (MLE) a 95\% threshold is drawn $\left(M L E+\chi_{0.95}^{2}\right)$ and the $95 \%$ confidence region is defined. For the parameter a the confidence interval ranges between 0.17 and 0.18 , which is in close agreement with $1 / \mu_{33}$. Also the parameters defining the measurement noise $\left(\sigma_{M}\right.$ and $\left.\sigma_{R H}\right)$ are estimated well. Based on the profile likelihood analysis all the parameters are found to be practically identifiable. Furthermore, the target values all fall in the $95 \%$ confidence interval. 
Postprint: Vereecken E, Roels S, Janssen H, 2018. Inverse hygric property determination based on dynamic measurements and swarm-intelligence optimisers, Building and Environment 131: 184-196.

https://doi.org/10.1016/j.buildenv.2017.12.030
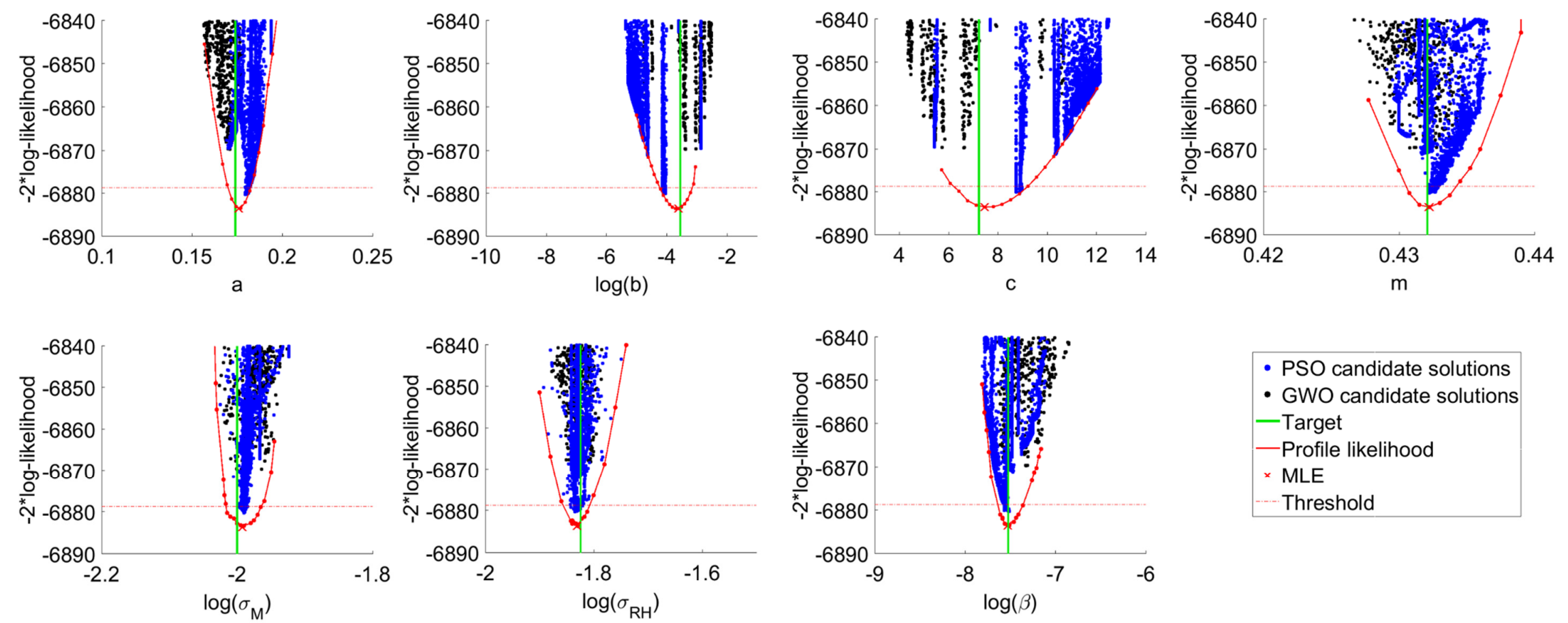

- PSO candidate solutions

- GWO candidate solutions

- Target

-Profile likelihood

$\times$ MLE

Threshold

Figure 9. Scatter plots of the candidate solutions analysed during the PSO and GWO process together with the profile

likelihood curve, the $95 \%$ threshold and the target values. 
Postprint: Vereecken E, Roels S, Janssen H, 2018. Inverse hygric property determination based on dynamic measurements and swarm-intelligence optimisers, Building and Environment 131: 184-196.

https://doi.org/10.1016/j.buildenv.2017.12.030

\subsection{Efficiency assessment}

An important criterion to decide on the most appropriate optimisation algorithm for the optimisation problem is the optimiser's efficiency, which indicates how fast the desired optimum is reached. In the current study, the objective function evaluations require solving the partial differential equation for moisture transport, which has the largest share in the total computational time. Therefore, in what follows the efficiency is analysed based on the number of objective function evaluations needed to reach an optimum. If an optimum is reached can be investigated based on, for example, the convergence of the likelihood (or cost) described by the objective function or - if the target values are known - by the convergence towards the target values. In this study, the latter approach is followed.

Figure 10 compares the efficiency of the PSO and GWO with two other popular optimisers: the Genetic Algorithm [20] and the Covariance Matrix Adaptation Evolution Strategy $[23,69]$. To this end the convergence of the estimations towards the target values is given as a function of the number of objective function evaluations. For this analysis again only the best run of each set of 10 runs is taken into account. Since the values for the parameter $m(\mathrm{Eq}(12))$ and the parameters $a, b$, and $c(\mathrm{Eq} .(13))$ applied to create the synthetic data are not an end in itself, the efficiency of the optimisation algorithms is evaluated based on physical properties; i.e. $\mu_{33}, \mu_{75}, \mu_{85}, w_{33}, w_{75}$ and $w_{85}$. The population size in the different optimisers is set to 10 and thus equal to the swarm size/number of search agents used in the PSO and GWO. The other settings applied in these optimisers are reported in Table 2. The convergence is analysed based on the logarithm of the difference between the best solution during the optimisation process 
Postprint: Vereecken E, Roels S, Janssen H, 2018. Inverse hygric property determination based on dynamic measurements and swarm-intelligence optimisers, Building and Environment 131: 184-196.

https://doi.org/10.1016/j.buildenv.2017.12.030

and the target value, averaged over the 10 selected runs. A lower value indicates a better agreement with the target values. As a reference, the $1 \%, 5 \%$ and $10 \%$ error on the target values is indicated by the dotted lines.

The GWO and the PSO (as well as the CMA-ES) are found to outperform the standard Genetic Algorithm (GA), which seems to reach local minima and does hence not succeed in estimating the target values well. For the PSO, a steep decline of the curves during the first 100 iterations is clearly visible. The PSO reveals to reach an optimum in a more efficient way in comparison to the other optimisers. The GWO reaches, for a maximum number of iterations equal to 400 (which is equal to 4000 objective function evaluations), the target values in a less efficient way in comparison to PSO and CMAES. This is especially visible for $\mu_{75}$ and $\mu_{85}$. By use of the GWO, however, for $\mu_{85}$ an error around $5 \%$ is obtained, while with PSO and CMA-ES this error is around $10 \%$. On the other hand, CMA-ES and PSO result in a better agreement with the target values for the moisture content. Though, the $1 \%$ error (or lower for $w_{33}, w_{75}$ ) as obtained by the GWO is already an extremely good result.

As the parameter estimation process with CMA-ES and PSO (and GA) could be stopped based on a convergence criterion, an additional comparison is made in which the number of iterations applied in the GWO is reduced. When the maximum number of iterations is set to 300 , the efficiency of the GWO is improved. A reduction to 200 iterations makes the GWO equally or even slightly more efficient than CMA-ES. Moreover, the GWO gives a (slightly) better final result. This final outcome is also in close agreement with the results obtained after 200 iterations with PSO. The curves obtained with the GWO, however, show a strongly decreasing trend at the end of the 
Postprint: Vereecken E, Roels S, Janssen H, 2018. Inverse hygric property determination based on dynamic measurements and swarm-intelligence optimisers, Building and Environment 131: 184-196.

https://doi.org/10.1016/j.buildenv.2017.12.030 optimisation process, while for the other optimisers the curves are found to be converged. This decreasing trend makes that with the GWO an even better solution might still be possible when a further exploitation is performed.

Recall also the intrinsic property of the GWO that puts the focus on the exploration of the search space during the first half of the iteration process. This property was implemented in the GWO to reduce the risk of trapping in a local minimum but can have a negative impact on the efficiency of the optimisation algorithm. A recent study [70] suggests a modified Grey Wolf Optimiser (mGWO) in which an exponential instead of a linear decrease is applied for the values of $\boldsymbol{a}$ (see Eq.(7)) in the GWO. In this way, an improved balance between exploration and exploitation is pursued. Though, in the current study this was found to have only a minor impact (not shown). The choice of the final number of iterations has a far stronger impact.

Table 2. Settings used in the optimisers.

\begin{tabular}{|l|l|}
\hline Optimiser & Settings \\
\hline PSO & $\begin{array}{l}\text { Maximum number of iterations }=400 \\
\text { Swarm size }=10 \\
\text { Personal learning coefficient } c_{1}=1.5, \\
\text { Global learning coefficient } c_{2}=2, \\
\text { Inertia weight } w=1, \\
\text { Inertia weight damping ratio } w_{\text {damp }}=0.99 . \\
\text { Minimum velocity }=-0.1 \\
\text { Maximum velocity }=0.1\end{array}$ \\
\hline GWO & $\begin{array}{l}\text { Maximum number of iterations }=400 \\
\text { Number of search agents }=10\end{array}$ \\
\hline
\end{tabular}




\begin{tabular}{|c|c|}
\hline GA & $\begin{array}{l}\text { Maximum number of iterations }=400 \\
\text { Population size } n_{\text {pop }}=10 \\
\text { Crossover } \\
\quad \text { Crossover percentage } p_{c}=0.7 \\
\quad \text { Number of offsprings } / \text { parents } n_{c}=2 \cdot \text { round }\left(p_{c} \cdot n_{p o p} / 2\right) \\
\quad \text { Extra range factor for crossover } \mathrm{Y}=0.4 \\
\text { Mutation } \\
\quad \text { Mutation percentage } p_{m}=0.3 \\
\quad \text { Number of mutants } n_{m}=\text { round }\left(p_{m} \cdot n_{p o p}\right) \\
\quad \text { Mutation rate } \mu=0.1 \\
\text { Roulette wheel selection with selection pressure } \beta=8\end{array}$ \\
\hline CMA & 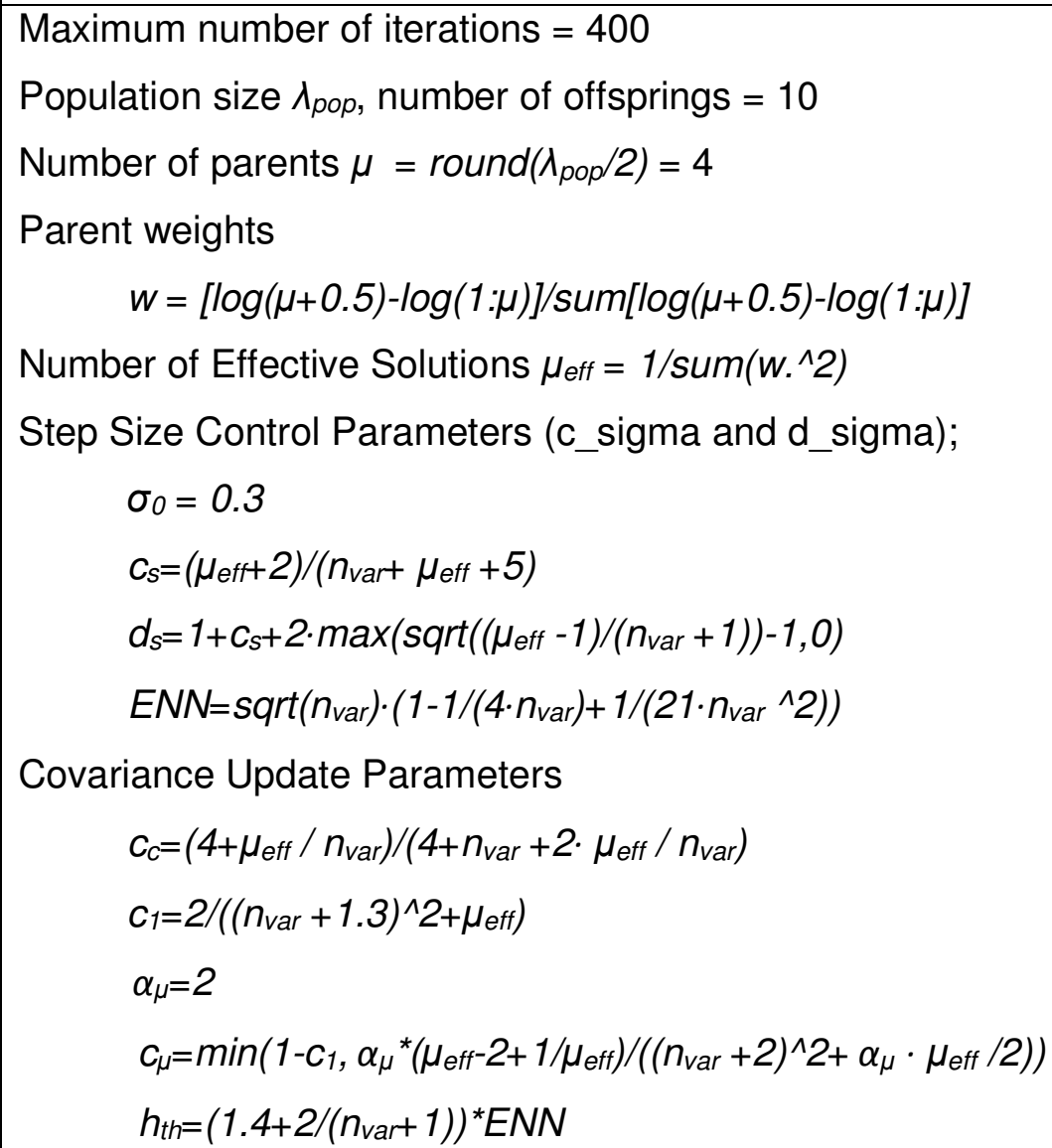 \\
\hline
\end{tabular}


Postprint: Vereecken E, Roels S, Janssen H, 2018. Inverse hygric property determination based on dynamic measurements and swarm-intelligence optimisers, Building and Environment 131: 184-196.
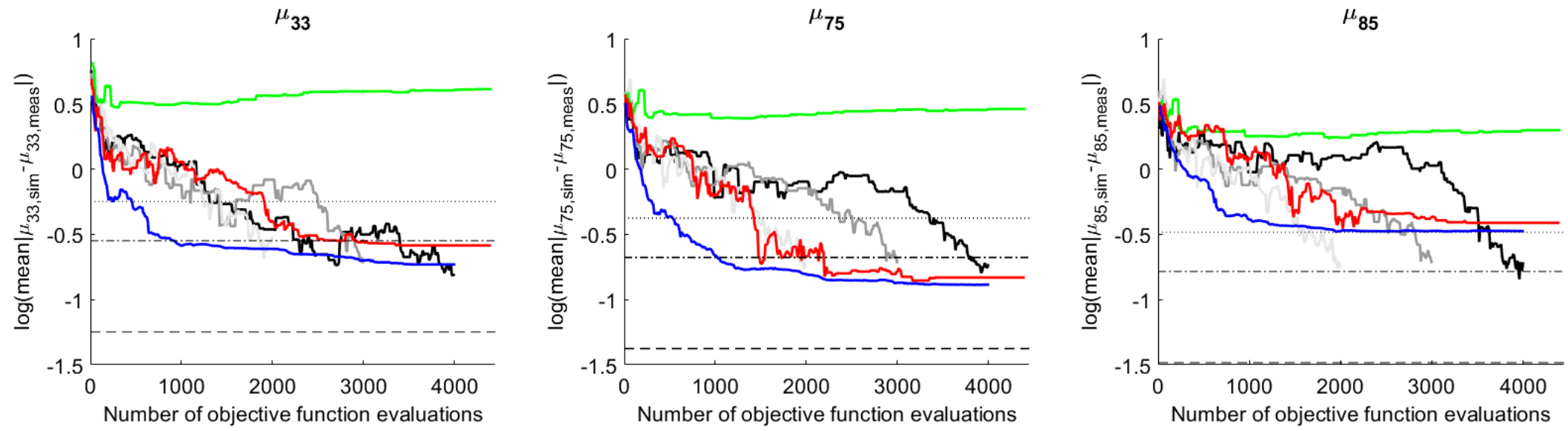

\begin{tabular}{|l}
\hline -GWO \\
- GWO (it 300) \\
GWO (it 200) \\
- GA \\
\hline CMA-ES \\
- PSO \\
$10 \%$ error \\
$---5 \%$ error \\
$---1 \%$ error
\end{tabular}
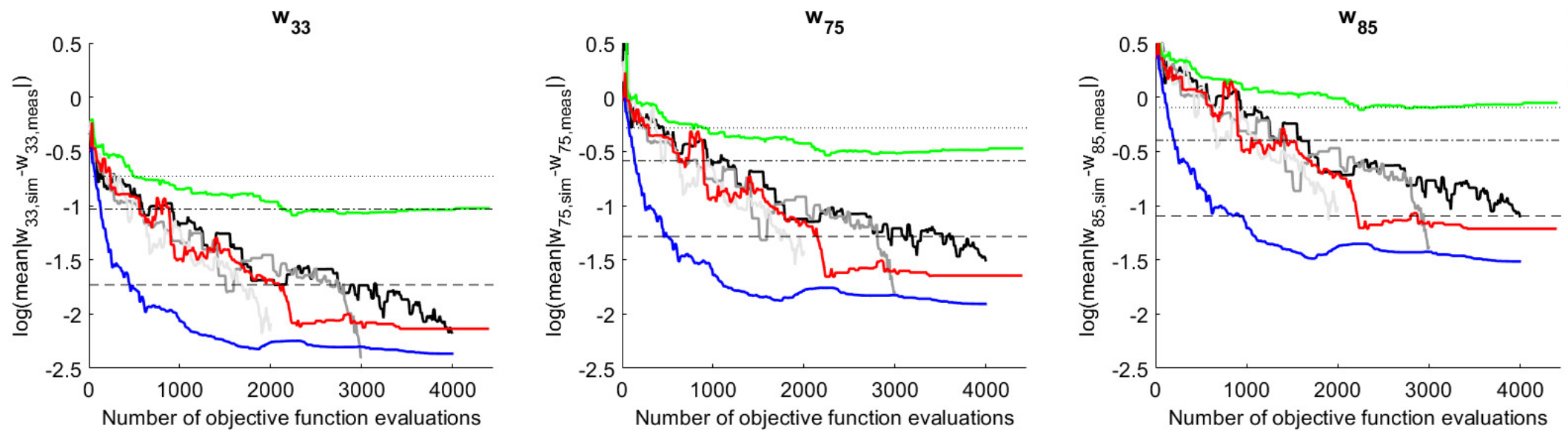

Figure 10. Comparison of the efficiency of the PSO and GWO to other popular optimisers. 
Postprint: Vereecken E, Roels S, Janssen H, 2018. Inverse hygric property determination based on dynamic measurements and swarm-intelligence optimisers, Building and Environment 131: 184-196.

https://doi.org/10.1016/j.buildenv.2017.12.030

\subsection{Robustness assessment}

Apart from the efficiency, a good optimisation algorithm should be able to reach the same optimum when starting from different initial candidate solutions. This performance is called the robustness. In the current study, the robustness of the PSO and GWO is studied based on the logarithm of the variance of the properties estimated in the 10 best runs each of them selected out of a set of 10 independent runs, as shown in Figure 11. A lower variance indicates that in the 10 selected runs convergence to a same value occurs, hence denoting a more robust optimiser. The robustness of the results obtained at the end of the optimisation process are of main importance and is shown to be very good for the PSO and the GWO. The lower robustness of the GWO during the initial iterations in the optimisation process is again a consequence of the focus on exploration during the first half of the iteration; though, this phenomenon is not important for the reliability of the results.

In addition to the convergence to a same optimum, an important feature of the optimisation algorithm is its capacity to converge to a global optimum. The latter should be taken into account when analyzing the robustness. Thereto, Figure $12 \mathrm{a}$ shows the likelihood (indicated by two times the log-likelihood) for the 100 independent runs, and this ranked by the likelihood as suggested by Raue et al. [68]. A plateau in the curve indicates a local optimum. The lowest plateau in the curve is assumed to be the global optimum (though as shown in the profile likelihood analysis a parameter set with a slightly better likelihood can be found). For the GWO and the PSO clear plateaus can be distinguished, indicating several runs that reach the same optimum. For the CMAES the plateaus are not clearly represented; though this could be partially due to the 
Postprint: Vereecken E, Roels S, Janssen H, 2018. Inverse hygric property determination based on dynamic measurements and swarm-intelligence optimisers, Building and Environment 131: 184-196.

https://doi.org/10.1016/j.buildenv.2017.12.030

limited number of iterations in the current study and the lack of convergence for some of the cases. The lower likelihood (in the figure plotted as the higher negative of two times the log-likelihood) together with the lower reproducibility of the same likelihood indicate a lower performance of the CMA-ES. For the GA the lower likelihood obtained in the different runs is clearly visible.

To get a view on the material properties estimated in the different runs, Figure $12 \mathrm{~b}, \mathrm{c}$, $d$ and e show these properties for the four likelihood ranges obtained based on the GWO (Figure 12a). The properties estimated in the 43 best runs are in good agreement with the target properties. The runs with a slightly lower likelihood delivered a nonphysically plausible vapour diffusion resistance in the higher relative humidity range. The remaining runs resulted in a non-plausible vapour diffusion resistance over the full relative humidity range. This again shows that despite the exploration and exploitation strategy embedded in the GWO - or the other strategies implemented in other optimisers - it is strongly recommended to perform a number of parameter estimation runs followed by the selection of the run with the highest likelihood. Furthermore, the reliability of the parameter estimation could benefit from a further implementation of restriction strategies to exclude physically non-plausible estimations. A forward analysis with the estimated properties as an input shows that also the physically nonplausible properties result in a good agreement with the measured output (Figure 13). 
Postprint: Vereecken E, Roels S, Janssen H, 2018. Inverse hygric property determination based on dynamic measurements and swarm-intelligence optimisers, Building and Environment 131: 184-196.
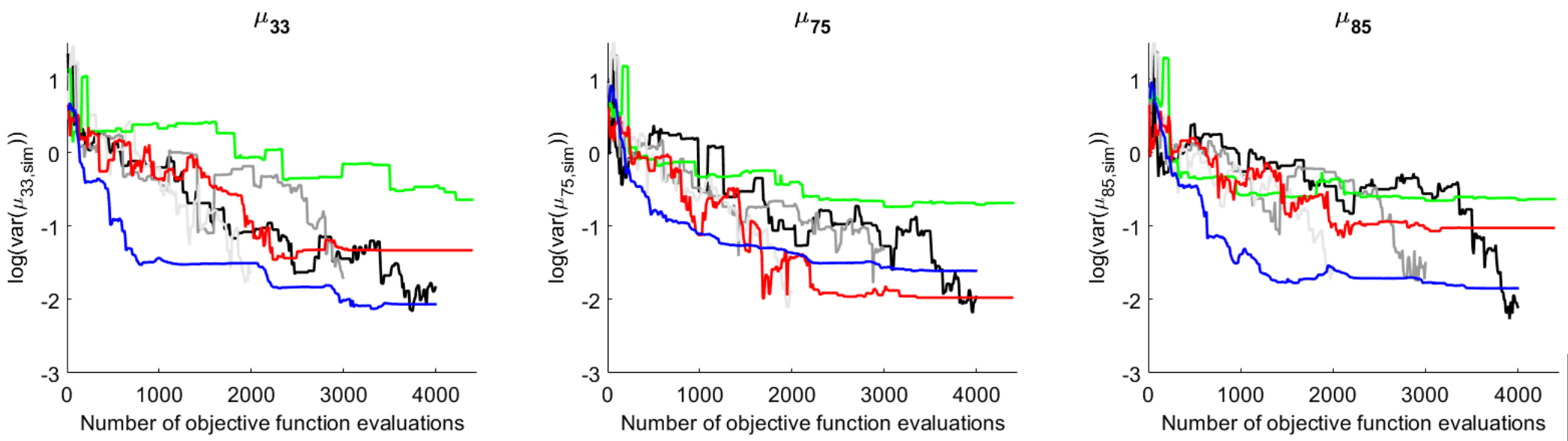

- GWO (it 300)
GWO (it 200)
GA
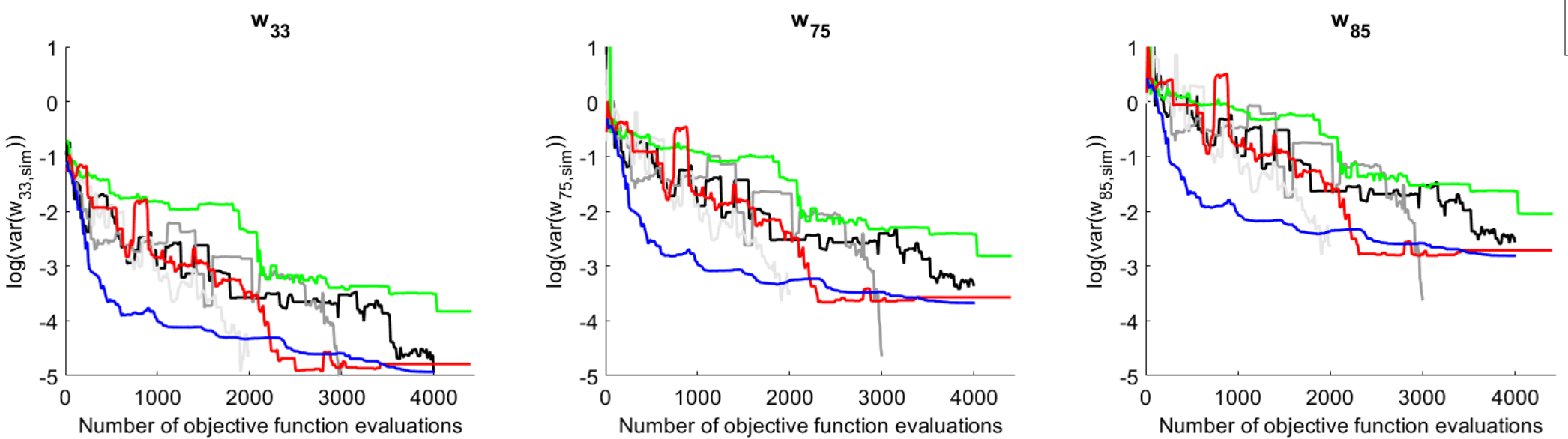

Figure 11. Comparison of the robustness of the PSO and GWO to other popular optimisers. 
Postprint: Vereecken E, Roels S, Janssen H, 2018. Inverse hygric property determination based on dynamic measurements and swarm-intelligence optimisers, Building and Environment 131: 184-196.

(c)

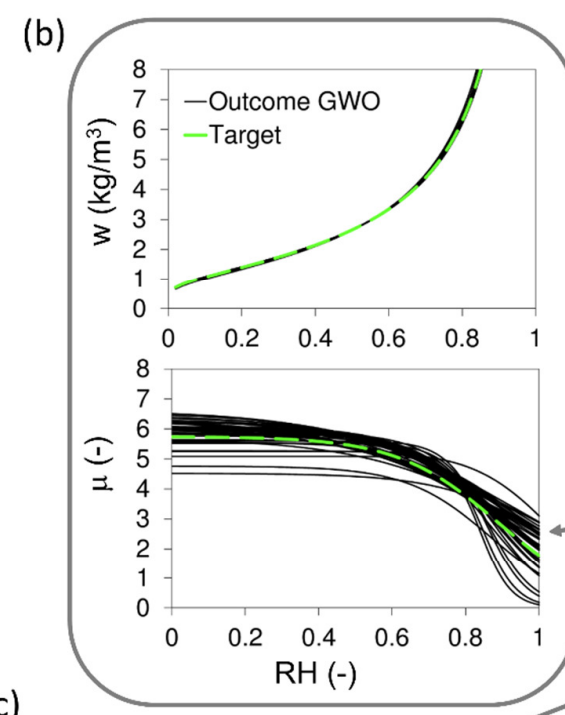

(a)
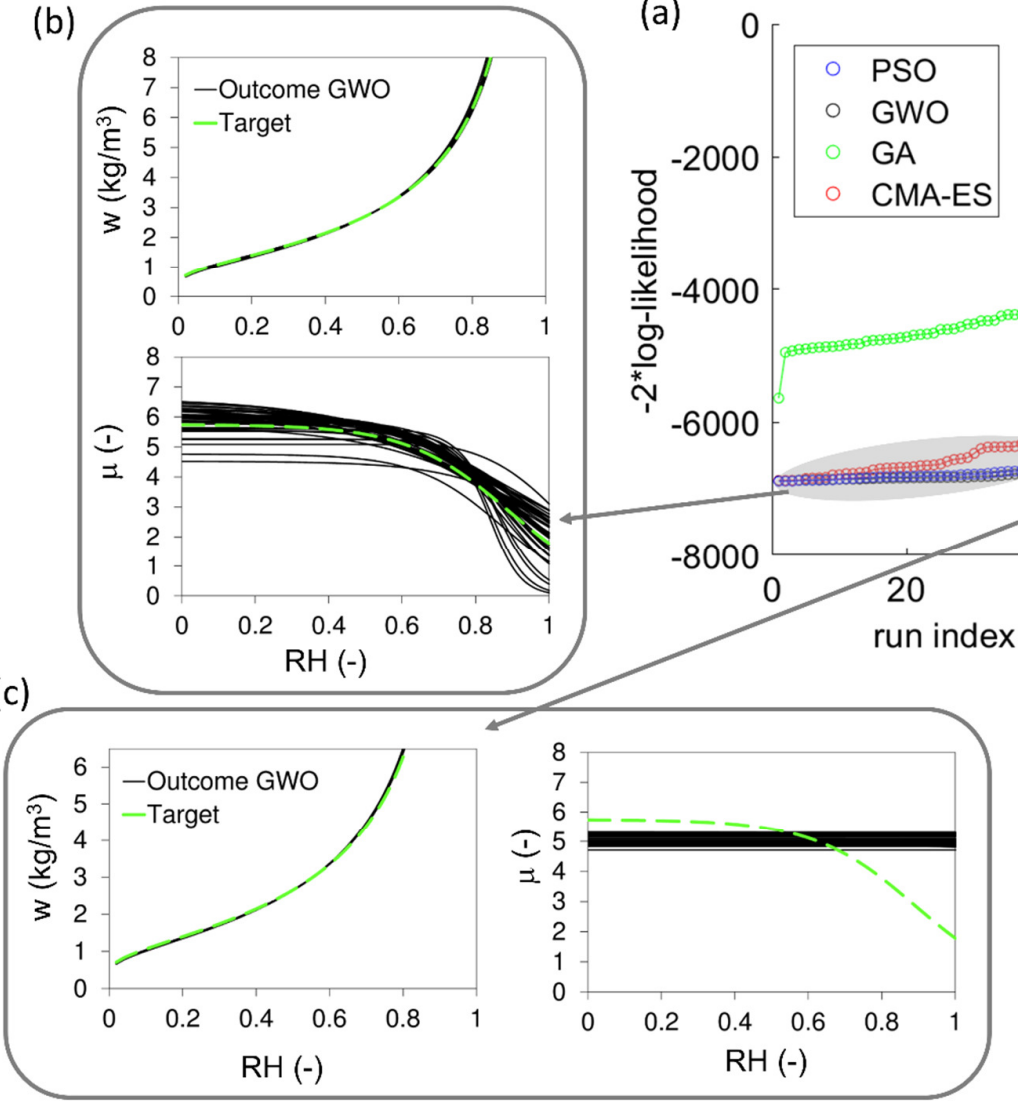

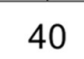

40

(d)

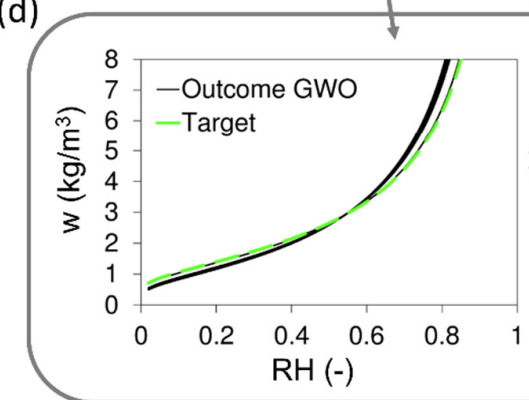

(e)
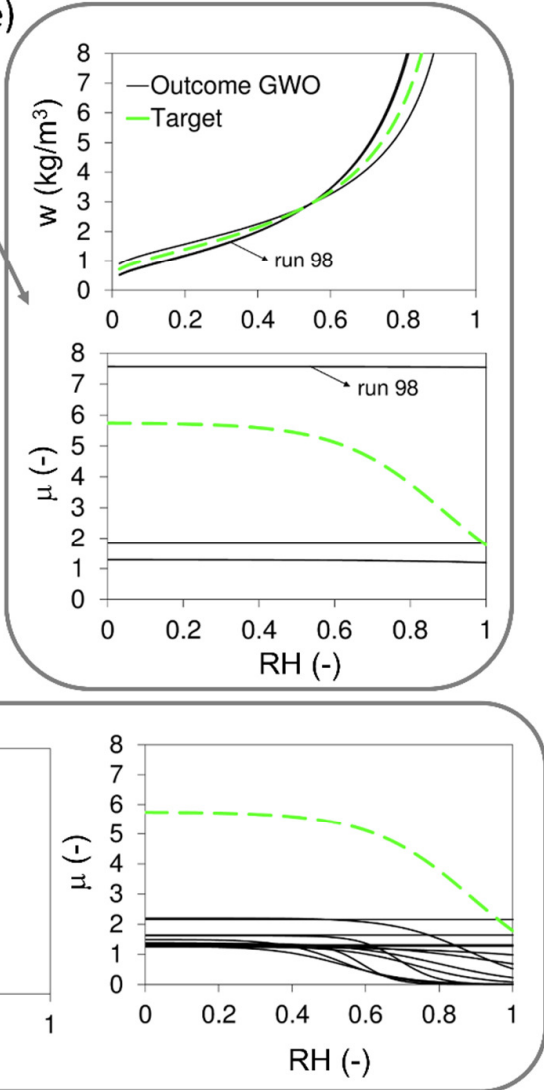

Figure 12. (a) Performance analysis based on the ranked likelihood values and $(b, c, d, e)$ the material properties estimated in the different runs with the PSO. 
(a)

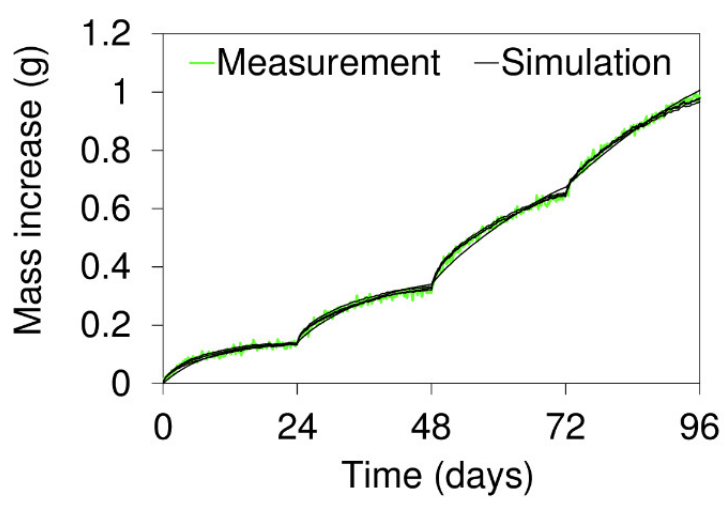

(b)

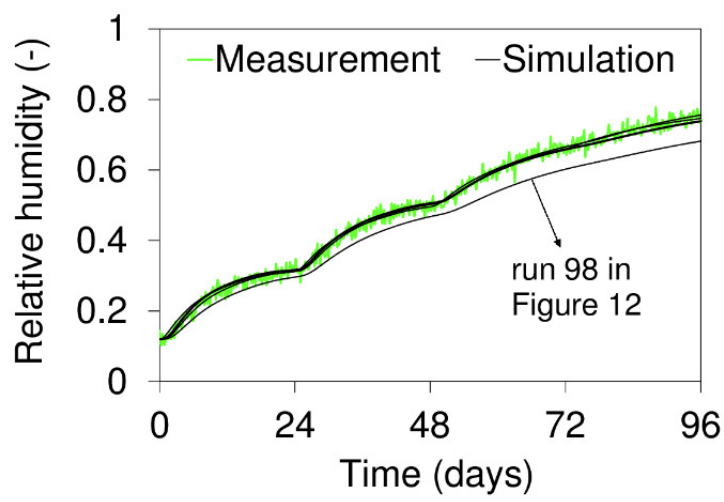

Figure 13. Comparison between synthetic experimental outcome ('Measurement') and estimated outcome ('Simulation'): (a) mass increase and (b) relative humidity measured at the bottom of the sample.

\section{Discussion and conclusions}

This paper presents a straightforward dynamic sorption experiment that allows the determination of the building material's sorption isotherm and vapour resistance factor as a function of relative humidity, and this in a much faster way than feasible by the standard steady-state tests. To infer the hygric properties from the dynamic measurement output two swarm-intelligence optimisers, i.e. the Particle Swarm Optimiser (PSO) and the Grey Wolf Optimiser (GWO) are applied. The applicability is assessed for synthetic data enriched with potential measurement noise. For both optimisers, the estimated sorption isotherm as well as the vapour resistance factor are found to be in close agreement with the target properties. It should however be kept in mind that the estimated parametric functions are not valid outside the relative humidity 
Postprint: Vereecken E, Roels S, Janssen H, 2018. Inverse hygric property determination based on dynamic measurements and swarm-intelligence optimisers, Building and Environment 131: 184-196.

https://doi.org/10.1016/j.buildenv.2017.12.030

range applied in the experimental test (in this case $12 \%$ till $85 \%$ ). In addition to the hygric properties, also the noise level on the measurements was included in the parameter estimation and was estimated with a good agreement. This estimation of the noise level is important if one wants to estimate the material properties and was possible due to the way the noise level is included in the likelihood formulation. An analysis on the impact of system noise was out of the scope of the current study. Furthermore, based on 100 independent parameter estimation runs, it was shown that is not recommended to rely on a single parameter estimation run. By performing a set of runs and selecting the best one, the reliability can be improved. Additionally, a profile likelihood analysis was shown to be an interesting tool to assess the confidence of the parameter estimation. In the current study, the information already obtained in the parameter estimation process was used to choose the range for additional reoptimisations. The parameters were found to be practically identifiable. Furthermore, by performing the profile likelihood analysis a parameter combination with a slightly better likelihood was detected.

For the current case, the swarm-intelligence optimisers (PSO and GWO) outperformed the Genetic Algorithm (GA) and - in less extent - the Covariance Matrix Adaptation Evolution Strategy (CMA-ES). The Particle Swarm Optimiser (PSO) is preferred for its efficiency. On the other hand, the GWO has fewer settings - i.e. the number of search agents and the maximal number of iterations - and is easy to implement, while its performance is very acceptable. One drawback of the GWO in its current form is the need to choose the final number of iterations beforehand. This choice can have an impact on the efficiency. Modifications to the GWO, such as the implementation of an 
Postprint: Vereecken E, Roels S, Janssen H, 2018. Inverse hygric property determination based on dynamic measurements and swarm-intelligence optimisers, Building and Environment 131: 184-196.

https://doi.org/10.1016/j.buildenv.2017.12.030

embedded convergence criterion, can of course be made. But, in this way additional meta-parameters would be introduced and the simplicity of original GWO could get lost.

Furthermore, it is important to keep in mind that the current comparison is made for specific settings of the meta-parameters in the optimisation algorithms. These settings can have an impact on the outcome. By carefully tuning the meta-parameters of the GA and the CMA-ES, the performance of these optimisers could be improved. However, such a tuning process can be time-consuming. For the GWO and the PSO, in the current study, no tuning of the meta-parameters was needed to achieve a good performance. Furthermore, if one wants to improve the performance of the GWO and the PSO even more, choosing the optimal settings for the meta-parameters in these optimisation algorithms will be - due to the fewer and more straightforward metaparameters - easier compared to the GA and CMA-ES. Moreover, for the PSO a number of suggestions in respect to the settings of the meta-parameters can be found in the literature [27]. Finally, the findings in respect to the performance of the algorithms may not be extrapolated to other studies, keeping in mind the No Free Lunch (NFL) theorem [51].

\section{Acknowledgements}

Evy Vereecken is a postdoctoral fellow of the Research Foundation (FWO) - Flanders, Belgium (FWO project 12J5216N). This financial support is gratefully acknowledged. 
Postprint: Vereecken E, Roels S, Janssen H, 2018. Inverse hygric property determination based on dynamic measurements and swarm-intelligence optimisers, Building and Environment 131: 184-196.

https://doi.org/10.1016/j.buildenv.2017.12.030

\section{References}

[1] Langmans J, Klein R, Roels S (2012) Hygrothermal risks of using exterior air barrier systems for highly insulated light weight walls: A laboratory investigation. Build. Environ. 56:192-202.

[2] Van Belleghem M, Steeman M, Janssens A, De Paepe M (2014) Heat, air and moisture transport modelling in ventilated cavity walls. J. Build. Phys. 38(4):317349.

[3] Vereecken E, Van Gelder L, Janssen H, Roels S (2015) Interior insulation for wall retrofitting - A probabilistic analysis of energy savings and hygrothermal risks. Energy Build. 89:231-244.

[4] Simonson CJ, Salonvaara M, Ojanen T (2002) The effect of structures on indoor humidity - possibility to improve comfort and perceived air quality. Indoor Air $12: 243-251$.

[5] Hameury S, Lundström T (2004) Contribution of indoor exposed massive wood to a good indoor climate: in situ measurement campaign . Energy Build. 36:281-292.

[6] Vereecken E, Roels S, Janssen H (2011) In situ determination of the moisture buffer potential of room enclosures. J. Build. Phys. 34(3):223-246.

[7] Roels S, Carmeliet J, Hens H, Adan O, Brocken H, Černy R, et al. (2004) Interlaboratory comparison of hygric properties of porous building materials. J. Therm. Envel. Build. Sci. 27:307-325.

[8] Kuishan L, Xu Z, Jun G (2009) Experimental investigation of hygrothermal parameters of building materials under isothermal conditions. J. Build. Phys. 32:355-370. 
Postprint: Vereecken E, Roels S, Janssen H, 2018. Inverse hygric property determination based on dynamic measurements and swarm-intelligence optimisers, Building and Environment 131: 184-196.

https://doi.org/10.1016/j.buildenv.2017.12.030

[9] Wadsö L (1994) A test of different methods to evaluate the diffusivity from unsteady-state sorption measurements. Dry. Technol. 12(8):1863-1876.

[10] Arfvidsson J, Cunningham M (2000) A transient technique for determining diffusion coefficients in hygroscopic materials. Build. Environ. 35:239-249.

[11] Drchalová J, Černy R (2003) A simple gravimetric method for determining the moisture diffusivity of building materials. Constr. Build. Mater. 17:223-228.

[12] Anderberg A, Wadsö L (2008) Method for simultaneous determination of sorption isotherms and diffusivity of cement-based materials. Cement Concrete Res. 38: 89-94.

[13] Delgado J, Ramos N, de Freitas V (2011a) Application of hybrid and moment methods to the measurement of moisture diffusion coefficients of building materials. Heat Mass Transfer 47:1491-1498.

[14] Delgado JMPQ, Ramos NMM, de Freitas VP (2011b) Application of different transient sorption methods to evaluate moisture diffusion coefficients of building materials on the hygroscopic range. J. Build. Phys. 35(3):251-266.

[15] Pavik Z, Žumár J, Pavliková M, Černy R (2011) A Boltzman transformation method for investigation of water vapor transport in building materials. J. Build. Phys. 35(3):213-223.

[16] Levenberg K (1944). A Method for the Solution of Certain Non-Linear Problems in Least Squares. Q. Appl. Math. 2:164-168

[17] Kang W, Lee YH, Chung WY, Xu HL (2009) Parameter estimation of moisture diffusivity in wood by an inverse method. J. Wood Sci. 55:83-90. 
Postprint: Vereecken E, Roels S, Janssen H, 2018. Inverse hygric property determination based on dynamic measurements and swarm-intelligence optimisers, Building and Environment 131: 184-196.

https://doi.org/10.1016/j.buildenv.2017.12.030

[18] Rouchier S, Foray G, Woloszyn M, Roux J (2012) Influence of diffuse damage on the water vapour permeability of fibre-reinforced mortar. Transport Porous Med. 93:543-559.

[19] Nassiopoulos A, Bourquin F (2013) On-site building walls characterization. Numer. Heat Tr. A. Appl. 63(3):179-200.

[20] Holland J (1992) Adaptation in Natural and Artificial Systems: an introductory analysis with applications to biology, control, and artificial intelligence. Cambridge, MA: MIT Press. ISBN 978-0262581110.

[21] Tang KS, Man KF, Kwong S, He Q (1996) Genetic algorithms and their applications. IEEE Sign. Proces. Mag. 13(6):22-37.

[22] Kočí J, Žumár J, Pavik Z, Černy R (2011) Application of genetic algorithm for determination of water vapor diffusion parameters of building materials. J. Build. Phys. 35(3):238-250.

[23] Hansen N (2006) The CMA evolution strategy: A comparing review. Towards a new Evolutionary Computation, p.75-102. Berlin, Springer.

[24] Rouchier S, Woloszyn M, Kedowide Y, Béjat T (2016) Identification of the hygrothermal properties of a building envelope material by the covariance matrix adaptation evolution strategy. J. Build. Perform. Simu. 9(1):101-114.

[25] Eberhart RC, Kennedy J (1995) A new optimizer using Particle Swarm Theory. In: Proc. Sixth International Symposium on Micro Machine and Human Science (Nagoya, Japan), IEEE Serv. Cent., Piscataway, NJ, 39-43.

[26] Poli R, Kennedy J, Blackwell T (2007) Particle swarm optimization, Swarm Intell. 1:33-57. 
Postprint: Vereecken E, Roels S, Janssen H, 2018. Inverse hygric property determination based on dynamic measurements and swarm-intelligence optimisers, Building and Environment 131: 184-196.

https://doi.org/10.1016/j.buildenv.2017.12.030

[27] Marini F, Walczak B (2015) Particle swarm optimization (PSO). A tutorial.

Chemom. Intell. Lab. Syst. 149:153-165.

[28] Akay B, Karaboga D (2012) Artificial Bee Colony algorithm for large-scale problems and engineering design optimization. J Intell. Manuf. 23:1001-1014.

[29] Karaboga D, Gorkemli B, Ozturk C., Karaboga N (2014) A comprehensive survey: Artificial Bee Colony (ABC) algorithm and applications. Artif. Intell. Rev. 42:21-57.

[30] Dorigo M, Maniezzo V, Colorni A (1996) The ant system: optimization by a colony of cooperating agents. IEEE Trans. Syst. Man Cybern. Part B 26(1):29-41.

[31] Yang XS. (2009) Firefly Algorithms for Multimodal Optimization. In: Watanabe O., Zeugmann T. (eds) Stochastic Algorithms: Foundations and Applications. SAGA 2009. Lecture Notes in Computer Science, vol 5792. Springer, Berlin, Heidelberg. p.169-178.

[32] Yang X (2010) A new metaheuristic Bat-inspired Algorithm. in: Nature Inspired Cooperative Strategies for Optimization (NICSO 2010) (Eds. J. R. Gonzalez et al.), SCl 284, 65-74,

[33] Yang X, Deb S (2010) Engineering Optimisation by Cuckoo Search. Int. J. Math. Mod. Num. Opt. 1(4):330-343.

[34] Yang X (2014) Cuckoo Search and Firefly Algorithm: Overview and analysis. In: Yan X (ed.) Cuckoo Search and Firefly Algorithm. Theory and Applications. p.1-26. Editor: Yang X. Springer, Cham. DOI 10.1007/978-3-319-02141-6.

[35] Can U, Alatas B (2015) Physics based metaheuristic algorithms for global optimization. Am. J. Inf. Sci. Comput. Eng. 1(3):99-106. 
Postprint: Vereecken E, Roels S, Janssen H, 2018. Inverse hygric property determination based on dynamic measurements and swarm-intelligence optimisers, Building and Environment 131: 184-196.

https://doi.org/10.1016/j.buildenv.2017.12.030

[36] Rashedi E, Nezamabadi-pour H, Saryazdi S (2009) GAS: A Gravitational

Search Algorithm. Inform. Sciences 179:2232-2248.

[37] Mahmoudi SM, Aghaie M, Bahonar M, Poursalehi N (2016) A novel optimization method, Gravitational Search Algorithm (GSA), for PWR core optimization. Ann. Nucl. Energy 95:23-34.

[38] Erol OK, Eksin I (2006) A new optimization method: Big Bang-Big Crunch. Adv. Eng. Softw. 37(2):106-111.

[39] Hosseini Z, Jafarian A (2016) A Hybrid Algorithm based on Invasive Weed Optimization and Particle Swarm Optimization for Global Optimization. Int. J. Adv. Comput. Sci. Appl. 7(10):295-303.

[40] Jadon SS, Tiwari R, Sharma H, Bansal JC (2017) Hybrid Artificial Bee Colony algorithm with Differential Evolution. Appl. Soft Comput. 58:11-24.

[41] Eberhart R, Shi Y (2001) Particle Swarm Optimization: Developments, Applications and Resources. Proceedings of the 2001 Congress on Evolutionary Computation, Seoul, South Korea, p.81-86.

[42] Hu X, Shi Y, Eberhart R (2004) Recent advances in particle swarm. Congress on Evolutionary Computation, Portland, OR, USA, p.90-97.

[43] Mirjalili S, Mirjalili SM, Lewis A (2014) Grey Wolf Optimizer. Adv. Eng. Softw. 69:46-61.

[44] Song X, Tang L, Zhao S, Zhang X, Li L, Huang J, Cai W (2015) Grey Wolf Optimizer for parameter estimation in surface waves. Soil Dyn. Earthq. Eng. 75:147-157. 
Postprint: Vereecken E, Roels S, Janssen H, 2018. Inverse hygric property determination based on dynamic measurements and swarm-intelligence optimisers, Building and Environment 131: 184-196.

https://doi.org/10.1016/j.buildenv.2017.12.030

[45] Komaki G.M., Kayvanfar V (2015) Grey Wolf Optimizer algorithm for the twostage assembly flow shop scheduling problem with release time. J. Comput. Sci. 8:109-120.

[46] Medjahed S, Ait Saadi T, Benyettou A, Ouali M (2016) Gray Wolf Optimizer for hyperspectral band selection. Appl. Soft Comput. 40:178-186.

[47] Sen Z, Zhou Y, Li Z, Pan W (2016) Grey wolf optimizer for unmanned combat aerial vehicle path planning. Adv. Eng. Softw. 99: 121-136.

[48] Pradhan M, Roy P, Pal T (2016) Grey Wolf Optimization applied to economic load dispatch problems. Elect. Pow. Energy Syst. 83:325-334.

[49] Jayabarathi T, Raghunathan T, Adarsh B.R., Suganthan P.N. (2016) Economic dispatch using hybric grey wolf optimizer. Energy 111:630-641.

[50] Jayakumar N, Subramanian S, Ganesan S, Elanchezhian E.B. (2016) Grey Wolf Optimization for combined heat and power dispatch with cogeneration systems. Electrical Poser and Energy Syst. 74:252-264.

[51] Wolpert D, Macready G (1997) No Free Lunch theorems for optimization. IEEE Trans. Evol. Comput. 1(4):67-82.

[52] Ho Y.C., Pepyne D.L. (2002) Simple explanation of the No-Free-Luunch Theorem and its implications. J. Optim. Theory Appl. 115(3):549-570.

[53] Ritter A, Hupet F, Muñoz-Carpena R, Lambot S, Vanclooster M (2003) Using inverse methods for estimating soil hydraulic properties from field data as an alternative to direct methods. Agr. Water Manage. 59:77-96. 
Postprint: Vereecken E, Roels S, Janssen H, 2018. Inverse hygric property determination based on dynamic measurements and swarm-intelligence optimisers, Building and Environment 131: 184-196.

https://doi.org/10.1016/j.buildenv.2017.12.030

[54] Ritter A, Muñoz-Carpena R, Regalado CM, Vanclooster M, Lambot S (2004) Analysis of alternative measurements strategies for the inverse optimization of the hydraulic properties of a volcanic soil. J. Hydrol. 295:124-139.

[55] Minasny B, Field DJ (2005) Estimating soil hydraulic properties and their uncertainty: the use of stochastic simulation in the inverse modelling of the evaporation method. Geoderma 126:277-290.

[56] Vaddadi P, Nakamura T, Singh RP (2003) Inverse analysis for transient moisture diffusion through fiber-reinforced composites. Acta Mater. 51:177-193.

[57] Calabrese L, Bozzoli F, Bochicchio G, Tessadri B, Vocale P, Rainieri S (2015) Parameter estimation approach to thermal characterization of intumescent fire retardant paints. J. Phys. Conf. Series 655, 012048.

[58] Kramer R, van Schijndel J, Schellen H (2013) Inverse modelling of simplified hygrothermal building models to predict and characterize indoor climates. Build. Environ. 68:87-99.

[59] Jiménez MJ (2016) Reliable building energy performance characterisation based on full scale dynamic measurements. Report of Subtask 3, part 1: Thermal performance characterization based on full scale testing - description of the common exercises and physical guidelines. International Energy Agency, EBC Annex 58. ISBN 9789460189876.

[60] Deconinck A, Roels S (2017) Is stochastic grey-box modelling suited for physical properties estimation of building components from on-site measurements? J. Build. Phys. 40(5):444-471. 
Postprint: Vereecken E, Roels S, Janssen H, 2018. Inverse hygric property determination based on dynamic measurements and swarm-intelligence optimisers, Building and Environment 131: 184-196.

https://doi.org/10.1016/j.buildenv.2017.12.030

[61] Dubois S, McGregor F, Evrard A, Heath A, Lebeau F (2014) An inverse modelling approach to estimate the hygric parameters of a clay-based masonry during a Moisture Buffer Value test. Build. Environ. 81: 192-203.

[62] Pazera M, Salonvaara M (2009) Examination of stability of boundary conditions in water vapor transmission tests. J. Build. Phys. 33:45-64.

[63] Feng C, Janssen H, Feng Y, Meng Q (2015) Hygric properties of porous building materials: Analysis of measurement repeatability and reproducibility. Build. Environ. 85: 160-172.

[64] Hansen N, Ostermeier A (2001) Complete Derandomized Self-Adaptation in Evolution Strategies. Evol. Comput. 9(2):159-195.

[65] Roels S, Carmeliet J, Hens H (2003) HAMSTAD WP1: Final report: Moisture transfer properties and materials characterization. KU Leuven, Belgium.

[66] Rouchier S, Busser T, Pailha M, Piot A, Woloszyn M (2017) Hygric characterization of wood fibre insulation under uncertainty with dynamic measurements and Markov Chain Monte-Carlo algorithm. Build. Environ. 114:129-139.

[67] Raue A, Kreutz C, Maiwald T, Bachmann J, Schilling M, Klingmüller U, Timmer $\mathrm{J}$ (2009) Structural and practical identifiability ananlysis of partially observed dynamical models and exploiting the profile likelihood. Bioinformatics 25(15):1923-1929.

[68] Raue A, Schilling M, Benchmann J, Matteson A, Schelks M, Kaschek D, Hug S, Kreutz C, et al. (2013) Lessons learned from quantitative dynamical modelling in systems biology. PLoS ONE 8(9):1:17. 
Postprint: Vereecken E, Roels S, Janssen H, 2018. Inverse hygric property determination based on dynamic measurements and swarm-intelligence optimisers, Building and Environment 131: 184-196.

https://doi.org/10.1016/j.buildenv.2017.12.030

[69] Hansen N, Müller SD, Koumoutsakos P (2003) Reducing the time complexity of the derandomized evolution strategy with covariance matrix adaptation (CMAES). Evol. Comput. 11(1):1-18.

[70] Mittal N, Singh U, Sing Sohi B (2016) Modified Grey Wolf Optimizer for global engineering optimization. Appl. Comput. Intell. Soft Comput. http://dx.doi.org/10.1155/2016/7950348 (16p). 